\title{
Critic-observer-based decentralized force/position approximate optimal control for modular and reconfigurable manipulators with uncertain environmental constraints
}

\author{
Yuanchun $\mathrm{Li}^{1} \cdot$ Xinye $\mathrm{Zhu}^{1,2} \cdot{\text { Tianjiao } \mathrm{An}^{1} \cdot \text { Bo Dong }}^{1}$
}

Received: 30 May 2021 / Accepted: 5 September 2021 / Published online: 29 September 2021

(c) The Author(s) 2021

\begin{abstract}
A critic-observer decentralized force/position approximate optimal control method is presented to address the joint trajectory and contacted force tracking problem of modular and reconfigurable manipulators (MRMs) with uncertain environmental constraints. The dynamic model of the MRM systems is formulated as an integration of joint subsystems via extensive state observer (ESO) associated with the effect of interconnected dynamic coupling (IDC). A radial basis function neural network (RBF-NN) is developed to deal with the IDC effects among the independent joint subsystems. Based on adaptive dynamic programming (ADP) approach and policy iteration (PI) algorithm, the Hamilton-Jacobi-Bellman (HJB) equation is approximately solved by establishing critic NN structure and then the approximated optimal control policy can be derived. The closed-loop manipulator system is proved to be asymptotic stable by using the Lyapunov theory. Finally, simulation results are provided to demonstrate the effectiveness and advantages of the proposed control method.
\end{abstract}

Keywords Modular robot manipulators · Adaptive dynamic programming · Force/position optimal control $\cdot$ Neural network

\section{Introduction}

Modular and reconfigurable manipulators (MRMs) have attracted attentions in manipulator community. Until now, MRMs possess widely applications, e.g. space explorations, medical assistance, etc. The appropriate control schemes are required to guarantee robustness as well as efficiency of MRMs facing complex and uncertain environmental constraints.

Force/position control, as a useful way to tackle contacting environment tasks of manipulators, have attracted extensive attentions in practices. Recently, force/position control

\footnotetext{
$凶$ Bo Dong

dongbo@ccut.edu.cn

Yuanchun Li

liyc@ccut.edu.cn

Xinye Zhu

zhuxinye1995@outlook.com

Tianjiao An

TianjiaoAn@hotmail.com

1 Department of Control Science and Engineering, Changchun University of Technology, Changchun 130012, China

2 Baicheng Normal University, Baicheng 137000, China
}

approaches have been divided into parallel force/position control [1], hybrid force/position control [2], impedance [3] etc. Hu et al. [4] addressed force/position control problem of the manipulator via sliding mode control. A novel estimator based on sparse Gaussian process regression is presented for approximating interaction force [5]. However, in the complex and extreme environments, it is an urgent problem to prove robustness of manipulator systems, and consider comprehensive optimization of tracking performance and energy consumption.

Optimal control method developed 6 decades ago by Pontryagin [6], Bellman [7], and many papers have been reported [8-11]. Recently, adaptive dynamic programming (ADP) have been used in discrete-time systems [12-14], continuous-time systems [15-17], data driven systems [1820], input/output constraints [21,22], uncertain external disturbance [23-25] as well as actuator failures [26], etc. Some investigations reported ADP-based manipulator systems. An ADP-based NN method was presented for manipulator based on learning [27]. An RL optimal control method was proposed to solve dynamic compensation in manipulator tasks [28]. The methods are centralized control which we have mentioned above. An significant property of MRMs is that the modules can adjust without control parameters variation

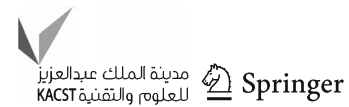


of other modules. Furthermore, local controllers need possess large storage capacity for calculating information under the centralized control policy. To solve above difficulties, decentralized control only utilizes the dynamic information and local states of each corresponding subsystem, which is considered an efficient method for MRMs. A network control method based on local information is presented by Jia [29]. An decentralized feedback control is presented for dead zone systems [30]. A decentralized method is developed for large-scale systems by Zhao [31]. A robust control method for reconfigurable manipulator is presented by Li [32]. Some researchers are proposed decentralized ADP-based method. Bian [33] developed a decentralized controller for unmatched uncertainties system. Zhao [34] developed a policy iteration (PI) for nonlinear systems. To the authors' point of view, there are few researches concentrated on dealing with optimal decentralized force/position control of manipulator systems, especially for MRM with uncertain environmental constraints.

Motivated by the researches above, a critic-observer decentralized force/position approximate optimal control method is developed to solve joint trajectory and contacted force tracking problem of MRMs with uncertain environmental constraints. The dynamic model of the MRM systems is formulated via extensive state observer (ESO) associated with interconnected dynamic coupling (IDC). A radial basis function neural network (RBF-NN) is developed to tackle IDC effects among the independent joint subsystems. Based on ADP and PI algorithms, the Hamilton-JacobiBellman (HJB) equation is approximately addressed using critic NN and then approximated optimal control policy can be derived. The closed-loop manipulator system is proved to be asymptotic stable. Simulation results are provided to show advantage of proposed control method.

The major contributions can be summarized as:

1. To the best of author's knowledge, it is first time to extend ADP to solve decentralized force/position control problem of MRMs with uncertain environmental constraints.

2. Because of different properties of the MRM dynamics, we present two compensator structure to deal with the robot system. ESO is proposed to solve unknown dynamic model because of different configurations and RBF-NN identifies the IDC effect among the modules. Through different compensate scheme, the tracking performance of MRM system can be improved by simulation verification.

The other part is organized as follows: Section "Problem statement and dynamic model" is problem statement and dynamic model formulation. The decentralized approximate optimal force/position control scheme is shown in section "Decentralized approximate optimal force/position control".
Simulations are verified the effectiveness of developed control method in section "Simulation". Section "Conclusion" presents conclusion.

\section{Problem statement and dynamic model}

Considering an MRM system is:

$M(q) \ddot{q}+C(q, \dot{q}) \dot{q}+G(q)+D=u+\tau_{c}$,

where $q \in R^{n}$ means joint movements, $M(q)$ denotes inertia matrix, $C(q, \dot{q})$ indicates centripetal Coriolis matrix, $G(q)$ means gravity term, $D$ denotes uncertain environment, $u \in R^{n}$ is control torque, $\tau_{c} \in R^{n}$ means constraint torque caused by end-effector contact with the environment, and there has $\tau_{c}=J_{\Phi}^{T}(q) \cdot f_{\text {for }}$, where $J_{\Phi}(q) \in R^{n \times n}$ means Jacobian matrix, $\Phi(q)$ is constraint equation, $f_{\text {for }}$ means constrained space force. The contact force is vertical to the motion method, which means the force will not change the directions.

Then, according to the reference [35], by separating terms depend on $\left(q_{i}, \dot{q}_{i}, \ddot{q}_{i}\right)$, each subsystem dynamic model is given as:

$M_{i}\left(q_{i}\right) \ddot{q}_{i}+C_{i}\left(q_{i}, \dot{q}_{i}\right) \dot{q}_{i}+G_{i}\left(q_{i}\right)+Z_{i}(q, \dot{q}, \ddot{q})+D_{i}=u_{i}+\tau_{c i}$,

where

$$
\begin{aligned}
Z_{i}= & \left(\sum_{j=1, j \neq i}^{n}\left(M_{i i}(q)-M_{i}\left(q_{i}\right)\right) \ddot{q}_{i}+M_{i j}(q) \ddot{q}_{j}\right) \\
& +\left(\sum_{j=1, j \neq i}^{n}\left(-C_{i}\left(q_{i}, \dot{q}_{i}\right)+C_{i i}(q, \dot{q})\right) \dot{q}_{i}+C_{i j}(q, \dot{q}) \dot{q}_{j}\right) \\
& +\left(\bar{G}_{i}(q)-G_{i}\left(q_{i}\right)\right),
\end{aligned}
$$

in which $q_{i}, \dot{q}_{i}, \ddot{q}_{i}, u_{i}, \tau_{c i}, D_{i}$ are $i$ th element of the vectors $q, \dot{q}, \ddot{q}, u, \tau_{c}, D ; M_{i}\left(q_{i}\right), C_{i}\left(q_{i}, \dot{q}_{i}\right), G_{i}\left(q_{i}\right)$ are $i$ th separate local element of $M(q), C(q, \dot{q}), G(q) ; Z_{i}$ is IDC term; $\bar{G}_{i}$ is the $i$ th element of $G ; M_{i j}, C_{i j}$ are $i j$ th element of $M$ and C.

Remark $1 D_{i}$ is considered as an uncertain environmental constraints term, that caused by the unmeasured environmental contact/collisions, rough surface of constraint, etc. The up-bound can be given as $\left|D_{i}\right| \leq T_{d M}$, where $T_{d M}$ means positive constant. In the two-link robot control case, the phenomenon of constraint is mainly resulting from the external environmental contact on the end-effector. 
Define the system state as $x_{i}=\left[x_{i 1}, x_{i 2}\right]^{T}=\left[q_{i}, \dot{q}_{i}\right]^{T}$, then, (2) can change as following

$$
\left\{\begin{array}{l}
\dot{x}_{i 1}=x_{i 2} \\
\dot{x}_{i 2}=f_{i}\left(x_{i 1}, x_{i 2}\right)+g_{i}\left(x_{i 1}\right)\left(u_{i}+\tau_{c i}\right)+h_{i}(x)+d_{i}, \\
y_{i}=x_{i 1}
\end{array}\right.
$$

in which the nonlinear terms $f_{i}\left(x_{i 1}, x_{i 2}\right), g_{i}\left(x_{i 1}\right), h_{i}(x)$ and $d_{i}$ are formulated as:

$$
\begin{aligned}
& f_{i}\left(x_{i 1}, x_{i 2}\right)=M_{i}^{-1}\left(q_{i}\right)\left(-C_{i}\left(q_{i}, \dot{q}_{i}\right) \dot{q}_{i}-G_{i}\left(q_{i}\right)\right) \\
& g_{i}\left(x_{i 1}\right)=M_{i}^{-1}\left(q_{i}\right) \\
& h_{i}(x)=-M_{i}^{-1}\left(q_{i}\right) Z_{i}(q, \dot{q}, \ddot{q}) \\
& d_{i}=-M_{i}^{-1}\left(q_{i}\right) D_{i},
\end{aligned}
$$

where $x$ is global state of the whole MRM and $f_{i}\left(x_{i 1}, x_{i 2}\right)$, $g_{i}\left(x_{i 1}\right)$ and $h_{i}(x)$ are all Lipschitz.

Next, a decentralized approximate optimal force/position control method is developed for MRM with uncertain environment to ensure trajectory tracking of MRM systems are asymptotically stable as well as the desired contact force can be satisfied.

\section{Decentralized approximate optimal force/position control}

\section{Derivation of the force/position optimal control}

For constrained MRM system (2), we can define the error function which is related to the trajectory tracking error as well as force tracking error:

$e_{i}(t)=k_{q w e i} \dot{e}_{q i}+k_{w e i} e_{q i}+k_{\tau i} \int_{0}^{t} e_{\tau i} \mathrm{~d} t$,

where $e_{q i}=x_{i 1}-x_{i 1 d}$ is position tracking error, $x_{i 1 d}$ means desired joint position, $e_{\tau i}=\tau_{c i}-\tau_{d i}$ is constrained torque deviation of end-effector, $\tau_{d i}$ means desired constraint torque, $\dot{e}_{q i}=\dot{x}_{i 1}-\dot{x}_{i 1 d}$ represents the velocity tracking error, $k_{q w e i}, k_{w e i}, k_{\tau i}$ are the function coefficients. The time derivative of $e_{i}$ is represented:

$\dot{e}_{i}(t)=k_{q w e i}\left(f_{i}\left(x_{i 1}, x_{i 2}\right)+g_{i}\left(x_{i 1}\right)\left(u_{i}+\tau_{c i}\right)+h_{i}(x)+d_{i}\right)+v_{i}$,

where $v_{i}=-k_{q w e i} \ddot{x}_{i 1 d}+k_{w e i} \dot{e}_{q i}+k_{\tau i} e_{\tau i}$.

Define performance index function as follows which is related to the error performance and control strategy: $\underset{u_{i} \in \Psi(\Omega)}{J_{i}\left(e_{i}\right)}=\int_{0}^{\infty}\left(\beta_{i} T_{d M}^{2}+N_{i}\left(e_{i}, u_{i}\right)+\Upsilon_{i}\left(\nabla J_{i}^{*}\left(e_{i}\right)\right)^{2}\right) d t$

where $T_{d M}$ is the known up-bound function for $d_{i}, N_{i}\left(e_{i}, u_{i}\right)=$ $e_{i}^{T} Q_{i} e_{i}+u_{i}^{T} R_{i} u_{i}$ means utility function composed of force/position deviation fusion, $N_{i}(0,0)=0$ as well as $N_{i}\left(e_{i}, u_{i}\right) \geq 0$ for all $e_{i}, u_{i} . Q_{i}, R_{i}$ mean positive definite matrices and $\Upsilon_{i}$ means determined constant. $\Psi(\Omega)$ means admissible control policies.

Definition 1 Consider system (2), $u_{i}$ is admissible for performance index (7) on $\Omega$, and $J_{i}\left(e_{i}\right)$ is finite, $\forall e_{i} \in \Omega$. Give admissible control $u_{i} \in Z(\Omega)$, where $Z(\Omega)$ means admissible control set, and infinite form of (8) is Lyapunov equation,

$$
\begin{aligned}
0= & N_{i}\left(e_{i}, u_{i}\right)+\Upsilon_{i}\left(\nabla J_{i}{ }^{*}\left(e_{i}\right)\right)^{2}+\beta_{i} T_{d M}^{2}+\left(\nabla J_{i}\left(e_{i}\right)\right)^{T} \cdot \dot{e}_{i} \\
= & N_{i}\left(e_{i}, u_{i}\right)+\Upsilon_{i}\left(\nabla J_{i}{ }^{*}\left(e_{i}\right)\right)^{2}+\beta_{i} T_{d M}^{2}+\left(\nabla J_{i}\left(e_{i}\right)\right)^{T} . \\
& \left(k_{\text {qwei }}\left(f_{i}\left(x_{i 1}, x_{i 2}\right)+g_{i}\left(x_{i 1}\right)\left(u_{i}+\tau_{c i}\right)+h_{i}(x)+d_{i}\right)+v_{i}\right),
\end{aligned}
$$

with $J_{i}^{*}\left(e_{i}\right)$ means optimal performance index function and $\nabla J_{i}\left(e_{i}\right)$ is partial derivative of $J_{i}\left(e_{i}\right)$ which has $\nabla J\left(e_{i}\right)=$ $\frac{\partial J\left(e_{i}\right)}{\partial e_{i}}$.

The HJB function of the force/position control under uncertain environmental constraints is:

$$
\begin{aligned}
H_{i}\left(e_{i}, u_{i}, \nabla J_{i}\left(e_{i}\right)\right) & =\beta_{i} T_{d M}^{2}+\Upsilon_{i}\left(\nabla J^{*}\left(e_{i}\right)\right)^{2}+N_{i}\left(e_{i}, u_{i}\right) \\
& +\left(\nabla J_{i}\left(e_{i}\right)\right)^{T} \cdot \dot{e}_{i}=\beta_{i} T_{d M}^{2}+e_{i}{ }^{T} Q_{i} e_{i}+u_{i}{ }^{T} R_{i} u_{i} \\
& +\Upsilon_{i}\left(\nabla J_{i}{ }^{*}\left(e_{i}\right)\right)^{2}+\left(\nabla J_{i}\left(e_{i}\right)\right)^{T} \cdot\left(k _ { q w e i } \left(f_{i}\left(x_{i 1}, x_{i 2}\right)\right.\right. \\
& \left.\left.+g_{i}\left(x_{i 1}\right)\left(u_{i}+\tau_{c i}\right)+h_{i}(x)+d_{i}\right)+v_{i}\right),
\end{aligned}
$$

and the optimal performance index function is:

$J_{i}{ }^{*}\left(e_{i}\right)=\min _{u_{i}} \int_{0}^{\infty}\left(\beta_{i} T_{d M}^{2}+N_{i}\left(e_{i}, u_{i}\right)+\Upsilon_{i}\left(\nabla J_{i}{ }^{*}\left(e_{i}\right)\right)^{2}\right) \mathrm{d} t$.

$J_{i}^{*}\left(e_{i}\right)$ satisfies HJB equation.

$0=\min _{u_{i}} H_{i}\left(e_{i}, u_{i}, \nabla J_{i}^{*}\left(e_{i}\right)\right)$,

where $\nabla J^{*}\left(e_{i}\right)=\frac{\partial J^{*}\left(e_{i}\right)}{\partial e_{i}}$, if $J_{i}{ }^{*}\left(e_{i}\right)$ is continuously differentiable, the optimal force/po- sition control is

$u_{i}^{*}=-\frac{1}{2} k_{q w e i} R_{i}^{-1} g_{i}^{T} \nabla J_{i}^{*}\left(e_{i}\right)$.

Dealing with dynamic identification of MRM, IDC term and uncertain environmental constraints, respectively, rewrite 
optimal control law $u_{i}{ }^{*}$ as $u_{i}{ }^{*}=u_{i 1}+u_{i 2}+u_{i 3}{ }^{*}$, so that HJB equation can be modified as:

$$
\begin{aligned}
0= & \beta_{i} T_{d M}^{2}+e_{i}^{T} Q_{i} e_{i}+u_{i}^{T} R_{i} u_{i}+\Upsilon_{i}\left(\nabla J_{i}{ }^{*}\left(e_{i}\right)\right)^{2}+\left(\nabla J_{i}\left(e_{i}\right)\right)^{T} . \\
& \left(k _ { q w e i } \left(f_{i}\left(x_{i 1}, x_{i 2}\right)+g_{i}\left(x_{i 1}\right)\left(u_{i 1}+u_{i 2}+u_{i 3}{ }^{*}+\tau_{c i}\right)\right.\right. \\
& \left.\left.+h_{i}(x)+d_{i}\right)+v_{i}\right) .
\end{aligned}
$$

\section{ESO-based dynamic identification of MRM}

As we all know that the dynamic model of MRM is hard to directly obtain, an ESO is proposed to approximate nonlinear term $f_{i}\left(x_{i 1}, x_{i 2}\right), g_{i}\left(x_{i 1}\right)$.

According to the state space (4), we consider the MRM system without the IDC effects and uncertainty environmental constraints. Based on the idea of ESO, (4) can be augmented as

$$
\left\{\begin{array}{l}
\dot{x}_{i 1}=x_{i 2} \\
\dot{x}_{i 2}=x_{i 3}+g_{i}\left(x_{i 1}\right)\left(u_{i 1}+\tau_{c i}\right) \\
\dot{x}_{i 3}=F_{i}\left(x_{i 1}, x_{i 2}\right) \\
y_{i}=x_{i 1}
\end{array},\right.
$$

where $F_{i}\left(x_{i 1}, x_{i 2}\right)=\dot{f}_{i}\left(x_{i 1}, x_{i 2}\right)$. The estimation error is defined as $e_{i o}=\left[\begin{array}{lll}e_{i o 1} & e_{i o 2} & e_{i o 3}\end{array}\right]^{T}=\left[\begin{array}{lll}\hat{x}_{i 1} & \hat{x}_{i 2} & \hat{x}_{i 3}\end{array}\right]^{T}-$ $\left[\begin{array}{lll}x_{i 1} & x_{i 2} & x_{i 3}\end{array}\right]^{T}$, then the ESO is constructed as

$$
\left\{\begin{array}{l}
\dot{\hat{x}}_{i 1}=\hat{x}_{i 2}-\beta_{i 01} \varphi\left(\hat{x}_{i 1}-x_{i 1}\right) \\
\dot{\hat{x}}_{i 2}=\hat{x}_{i 3}-\beta_{i 02} \varphi\left(\hat{x}_{i 1}-x_{i 1}\right)+\hat{g}_{i}\left(\hat{x}_{i 1}\right)\left(u_{i 1}+\tau_{c i}\right) \\
\dot{\hat{x}}_{i 3}=-\beta_{i 03} \varphi\left(\hat{x}_{i 1}-x_{i 1}\right)
\end{array}\right.
$$

where $\varphi$ means a function about the estimation error, $\beta_{i 0 j}(j=1,2,3)$ is adjustable observer gain. The estimation error dynamic can be expressed as

$$
\left\{\begin{array}{l}
\dot{e}_{i o 1}=e_{i o 2}-\beta_{i 01} \varphi\left(e_{i o 1}\right) \\
\dot{e}_{i o 2}=e_{i o 3}-\beta_{i 02} \varphi\left(e_{i o 1}\right) \\
\dot{e}_{i o 3}=-\beta_{i 03} \varphi\left(e_{i o 1}\right)-F_{i}\left(\hat{x}_{i 1}, \hat{x}_{i 2}\right)
\end{array} .\right.
$$

Motivated by finite-time stable system, $\varphi\left(e_{i o 1}\right)$ can be defined as follows:

$\varphi\left(e_{i o 1}\right)=-k_{i} \operatorname{sgn}\left(e_{i o 1}\right)\left|e_{i o 1}\right|^{\kappa_{i}}, \kappa_{i} \in(0,1)$.

$$
\left\{\begin{array}{l}
\dot{e}_{i o 1}=e_{i o 2}-\beta_{i 01}\left|e_{i o 1}\right|^{\frac{\kappa_{i}+1}{2}} \operatorname{sgn}\left(e_{i o 1}\right) \\
\dot{e}_{i o 2}=e_{i o 3}-\beta_{i 02}\left|e_{i o 1}\right|^{\frac{\kappa_{i}+1}{2}} \operatorname{sgn}\left(e_{i o 1}\right) \\
\dot{e}_{i o 3}=-\beta_{i 03}\left|e_{i o 1}\right|^{\kappa_{i}} \operatorname{sgn}\left(e_{i o 1}\right)-F_{i}\left(\hat{x}_{i 1}, \hat{x}_{i 2}\right)
\end{array} .\right.
$$

Theorem 1 Consider the dynamic system with $f_{i}\left(x_{i 1}, x_{i 2}\right)$, $g_{i}\left(x_{i 1}\right)$ in (4) and the dynamic system is formulated in (14) as well as the up-bound of $F_{i}\left(x_{i 1}, x_{i 2}\right)$ is $F_{i M}$. The ESO presents in (15) and there exist constants $\beta_{i 0 j}>0(j=$ $1,2,3)$ and $\left(\beta_{i 01} \beta_{i 02}\right) \frac{\kappa_{i}+1}{2}-\beta_{i 03}>0$ such that can ensure $\lim _{t \rightarrow \infty} e_{i o}(t)=0$.

Proof For the ESO in Eq. (18), define

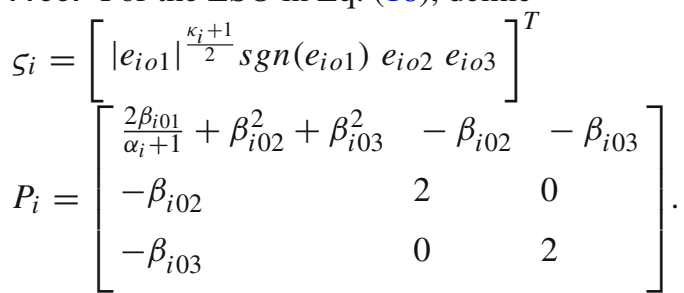

Then, the Lyapunov candidate function is chosen as

$V_{i}\left(\beta_{i 0 j}, \varsigma_{i}\right)=\varsigma_{i}^{T} P_{i} \varsigma_{i}$.

When $\beta_{i 0 j}>0, P_{i}$ is a symmetric positive definite matrix. Let $\lambda_{i}$ represents eigenvalue sequence,

$\lambda_{\min }\left(P_{i}\right)\left\|\varsigma_{i}\right\|_{2}^{2} \leq V_{i} \leq \lambda_{\max }\left(P_{i}\right)\left\|\varsigma_{i}\right\|_{2}^{2}$,

where $\left\|\varsigma_{i}\right\|_{2}^{2}=\left|e_{i o 1}\right|^{\kappa_{i}+1}+e_{i o 2}^{2}+e_{i o 3}^{2}$, and

$\left|e_{i o 1}\right|^{\frac{\kappa_{i}+1}{2}} \leq\left\|\varsigma_{i}\right\|_{2} \leq \sqrt{\frac{V_{i}}{\lambda_{i \min }\left(P_{i}\right)}}$.

To apply Lyapunov theorem, we derivative $V_{i}$ respect to time, thus we can obtain that

$$
\begin{aligned}
& \dot{V}_{i}=\left[\left|e_{i o 1}\right|^{\frac{k_{i}+1}{2}} \operatorname{sgn}\left(e_{i o 1}\right) e_{i o 2} e_{i o 3}\right] \\
& Y_{i}\left[\left|e_{i o 1}\right|^{\frac{\kappa_{i}+1}{2}} \operatorname{sgn}\left(e_{i o 1}\right) e_{i o 2} e_{i o 3}\right]^{T} \\
& +2 F_{i}\left(\beta_{i 03}\left|e_{i o 1}\right|^{\frac{\kappa_{i}+1}{2}} \operatorname{sgn}\left(e_{i o 1}\right)+2 e_{i o 3}\right) \\
& =-\varsigma_{i}^{T} Y_{i} \varsigma_{i}+2 F_{i}\left(\beta_{i 03}\left|e_{i o 1}\right|^{\frac{\kappa_{i}+1}{2}} \operatorname{sgn}\left(e_{i o 1}\right)+2 e_{i o 3}\right) \\
& =V_{i 1}+V_{i 2}
\end{aligned}
$$

where $Y_{i}$ can be defined as follows 


$$
Y_{i}=\left[\begin{array}{l}
-\beta_{i 01}\left(\kappa_{i}+1\right)\left(\frac{2 \beta_{i 01}}{\kappa_{i}+1}+\beta_{i 02}^{2}+\beta_{i 03}^{2}\right)\left|e_{i o 1}\right|^{\frac{\kappa_{i}-1}{2}}+2 \beta_{i 03}^{2}\left|e_{i o 1}\right|^{\frac{\kappa_{i}-1}{2}}+2 \beta_{i 02}^{2}, \\
\frac{\kappa_{i}+1}{2}\left|e_{i o 1}\right|^{\frac{\kappa_{i}-1}{2}}\left(\frac{2 \beta_{i 01}}{\kappa_{i}+1}+\beta_{i 02}^{2}+\beta_{i 03}^{2}+\beta_{i 01} \beta_{i 02}\right)-2 \beta_{i 02}, \beta_{i 01} \beta_{i 03} \frac{\kappa_{i}+1}{2} \\
-2 \beta_{i 03}\left|e_{i o 1}\right|^{\frac{\kappa_{i}-1}{2}}-\beta_{i 02} ; \frac{\kappa_{i}+1}{2}\left|e_{i o 1}\right|^{\frac{\kappa_{i}-1}{2}}\left(\frac{2 \beta_{i 01}}{\kappa_{i}+1}+\beta_{i 02}^{2}+\beta_{i 03}^{2}+\beta_{i 01} \beta_{i 02}\right) \\
-2 \beta_{i 02},-\beta_{i 02}\left(\kappa_{i}+1\right)\left|e_{i o 1}\right|^{\frac{\kappa_{i}-1}{2}},-\beta_{i 03} \frac{\kappa_{i}+1}{2}\left|e_{i o 1}\right|^{\frac{\kappa_{i}-1}{2}}+2, \beta_{i 01} \beta_{i 03} \\
\frac{\kappa_{i}+1}{2}\left|e_{i o 1}\right|^{\frac{\kappa_{i}-1}{2}} ;-2 \beta_{i 03}\left|e_{i o 1}\right|^{\frac{\kappa_{i}-1}{2}}-\beta_{i 02},-\beta_{i 03} \frac{\kappa_{i}+1}{2}\left|e_{i o 1}\right|^{\frac{\kappa_{i}-1}{2}}+2,0 ;
\end{array}\right],
$$

and can be equivalent to

$A_{i}^{T} P_{i}+P_{i} A_{i}=-Y_{i}$,

where

$$
\begin{aligned}
A_{i} & =\left[\begin{array}{lll}
-\beta_{i 01} \frac{\kappa_{i}+1}{2}\left|e_{i o 1}\right|^{\frac{\kappa_{i}-1}{2}} & \frac{\kappa_{i}+1}{2}\left|e_{i o 1}\right|^{\frac{\kappa_{i}-1}{2}} & 0 \\
-\beta_{i 02} & 1 \\
-\beta_{i 03}\left|e_{i o 1}\right|^{\frac{\kappa_{i}-1}{2}} & 0 & 0
\end{array}\right] \\
& =\left[\begin{array}{lll}
-\frac{\kappa_{i}+1}{2}\left|e_{i o 1}\right|^{\frac{\kappa_{i}-1}{2}} & 0 & 0 \\
0 & -1 & 0 \\
0 & 0 & -\left|e_{i o 1}\right|^{\frac{\kappa_{i}-1}{2}}
\end{array}\right]\left[\begin{array}{lll}
\beta_{i 01} & -1 & 0 \\
\beta_{i 02} & 0 & -1 \\
\beta_{i 03} & 0 & 0
\end{array}\right] .
\end{aligned}
$$

According to Eq. (24), we can obtain

$$
\begin{aligned}
\left|s I-A_{i}\right| & =\left|\begin{array}{ccc}
s+\beta_{i 01} \frac{\kappa_{i}+1}{2}\left|e_{i o 1}\right|^{\frac{\kappa_{i}-1}{2}} & -\frac{\kappa_{i}+1}{2}\left|e_{i o 1}\right|^{\frac{\kappa_{i}-1}{2}} & 0 \\
\beta_{i 02} & s & -1 \\
\beta_{i 03}\left|e_{i o 1}\right|^{\frac{\kappa_{i}-1}{2}} & 0 & s
\end{array}\right| \\
& =s^{3}+\beta_{i 01} \frac{\kappa_{i}+1}{2}\left|e_{i o 1}\right|^{\frac{\kappa_{i}-1}{2}} s^{2}+\beta_{i 02} \frac{\kappa_{i}+1}{2}\left|e_{i o 1}\right|^{\frac{\kappa_{i}-1}{2}} s \\
& +\beta_{i 03} \frac{\kappa_{i}+1}{2}\left|e_{i o 1}\right|^{\kappa_{i}-1} .
\end{aligned}
$$

From Eq. (25), the polynomial coefficients are all positive. If $\beta_{i 01}, \beta_{i 02}, \beta_{i 03}, \kappa_{i}$ are chosen properly, and satisfy

$$
\left\{\begin{aligned}
\beta_{i 01} \frac{\kappa_{i}+1}{2}\left|e_{i o 1}\right|^{\frac{\kappa_{i}-1}{2}}>0 \\
\beta_{i 02} \frac{\kappa_{i}+1}{2}\left|e_{i o 1}\right|^{\frac{\kappa_{i}-1}{2}}>0 \\
\beta_{i 03} \frac{\kappa_{i}+1}{2}\left|e_{i o 1}\right|^{\kappa_{i}-1}>0 \\
\beta_{i 01} \beta_{i 02} \frac{\kappa_{i}+1}{2}-\beta_{i 03}>0,
\end{aligned}\right.
$$

then $A_{i}$ is Hurwitz.

Remark 2 According to formulation of $A_{i}$ in (24), only guarantee $A_{i}$ is Hurwitz in (26), the observer can be effectiveness. First of all, we choose $\beta_{i 01}, \beta_{i 02}, \beta_{i 03}$ positive as well as , the first of three equations in (26) can be satisfied. That means if $\beta_{i 01} \beta_{i 02} \frac{\kappa_{i}+1}{2}>\beta_{i 03}$ satisfies, the parameters can choose properly.
According to the Lyapunov theory, there exists solution $P_{i}$ in Eq. (23).

$V_{i 1}=-\varsigma_{i}^{T} Y_{i} \varsigma_{i} \leq-\lambda_{\min }\left(Y_{i}\right)\left\|\varsigma_{i}\right\|_{2}^{2}$.

$V_{i 2}$ can be re-expressed as follows:

$$
\begin{aligned}
V_{i 2} & =2 F_{i}\left(\beta_{i 03}\left|e_{i o 1}\right|^{\frac{\kappa_{i}+1}{2}} \operatorname{sgn}\left(e_{i o 1}\right)+2 e_{i o 3}\right) \\
& =\left[\begin{array}{lll}
2 F_{i} & 0 & 4 F_{i}
\end{array}\right]\left[\begin{array}{c}
\left|e_{i o 1}\right|^{\frac{\kappa_{i}+1}{2}} \operatorname{sgn}\left(e_{i o 1}\right) \\
e_{i o 2} \\
e_{i o 3}
\end{array}\right] \\
& =\left[\begin{array}{lll}
2 F_{i} & 0 & 4 F_{i}
\end{array}\right] \varsigma_{i} \leq 2 \sqrt{5} F_{i M}\left\|\varsigma_{i}\right\|_{2} .
\end{aligned}
$$

Combing Eqs. (27), (28), we can know that

$$
\begin{aligned}
\dot{V}_{i} & =V_{i 1}+V_{i 2} \leq-\lambda \min \left(Y_{i}\right)\left\|\varsigma_{i}\right\|_{2}^{2}+2 \sqrt{5} F_{i M}\left\|\varsigma_{i}\right\|_{2} \\
& =-\left(\lambda_{\min }\left(Y_{i}\right)\left\|\varsigma_{i}\right\|_{2}-2 \sqrt{5} F_{i M}\right)\left\|\varsigma_{i}\right\|_{2} .
\end{aligned}
$$

$Y_{i}$ is positive definite and nonsingular, then

$$
\begin{aligned}
\lambda_{\min }\left(Y_{i}\right) & =\sigma_{\min }\left(Y_{i}\right)=\sigma_{\min }\left(-A_{i}^{T} P_{i}+P_{i} A_{i}\right) \\
& =\sigma_{\min }\left(\left(-A_{i}\right)^{T} P_{i}+\left(\left(-A_{i}\right)^{T} P_{i}\right)^{T}\right) \\
& =2 \sigma_{\min }\left(\left(-A_{i}\right)^{T} P_{i}\right) \\
& \geq 2 \sigma_{\min }\left(-A_{i}\right)^{T} \sigma_{\min }\left(P_{i}\right) \\
& =2 \min \left(\frac{\kappa_{i}+1}{2}\left|e_{i o 1}\right|^{\frac{\kappa_{i}-1}{2}}, 1\right) \sigma_{\min }\left(\Xi_{i}\right) \lambda_{\min }\left(P_{i}\right),
\end{aligned}
$$

where

$\left(-A_{i}\right)^{T}=\left[\begin{array}{lll}\frac{\kappa_{i}+1}{2}\left|e_{i o 1}\right|^{\frac{\kappa_{i}-1}{2}} & 0 & 0 \\ 0 & 1 & 0 \\ 0 & 0 & \left|e_{i o 1}\right|^{\frac{\kappa_{i}-1}{2}}\end{array}\right]\left[\begin{array}{lll}\beta_{i 01} & \beta_{i 02} & \beta_{i 03} \\ -1 & 0 & 0 \\ 0 & -1 & 0\end{array}\right]$ $=\Theta_{i} \Xi_{i}$.

When $\left|e_{i o 1}\right|>1,\left\|\varsigma_{i}\right\|_{2} \geq\left|e_{i o 1}\right|^{\frac{\kappa_{i}+1}{2}}, \lambda_{\min }\left(Y_{i}\right) \geq\left(\kappa_{i}+\right.$ 1) $\left|e_{i o 1}\right|^{\frac{\kappa_{i}-1}{2}} \sigma_{\min }\left(\Xi_{i}\right) \lambda_{\min }\left(P_{i}\right)$, thus 


$$
\begin{aligned}
& \lambda_{\min }\left(Y_{i}\right)\left\|s_{i}\right\|_{2}-2 \sqrt{5} F_{i M} \geq\left(\kappa_{i}+1\right) \sigma_{\min }\left(\Xi_{i}\right) \lambda_{\min }\left(P_{i}\right)-2 \sqrt{5} F_{i M} . \\
\dot{V} \leq- & \left(\lambda_{\min }\left(Y_{i}\right)\left\|\varsigma_{i}\right\|_{2}-2 \sqrt{5} F_{i M}\right)\left\|\varsigma_{i}\right\|_{2} \\
\leq & -\left(\left(\kappa_{i}+1\right) \sigma_{\min }\left(\Xi_{i}\right) \lambda_{\min }\left(P_{i}\right)-2 \sqrt{5} F_{i M}\right)\left\|\varsigma_{i}\right\|_{2} \\
\leq- & \left(\left(\kappa_{i}+1\right) \sigma_{\min }\left(\Xi_{i}\right) \lambda_{\min }\left(P_{i}\right)-2 \sqrt{5} F_{i M}\right)\left\|\varsigma_{i}\right\|_{2} \\
& \sqrt{\frac{V_{i}}{\lambda_{\max }\left(P_{i}\right)}} \\
= & \frac{\left(\left(\kappa_{i}+1\right) \sigma_{\min }\left(\Xi_{i}\right) \lambda_{\min }\left(P_{i}\right)-2 \sqrt{5} F_{i M}\right)}{\sqrt{\lambda_{\max }\left(P_{i}\right)}} V_{i}^{\frac{1}{2}} \leq 0 .
\end{aligned}
$$

This completes the proof of the Theorem 1 .

Remark 3 Different from the traditional ESO, the proposed ESO solves the problem of complicated parameter setting of the controller as well as reduce computation burden of MRM.

Therefore, $u_{i 1}$ can be defined as:

$u_{i 1}=k_{i p}\left(x_{i d}-\hat{x}_{i 1}\right)-k_{i d} \hat{x}_{i 2}-\hat{x}_{i 3}$,

where $k_{i p}, k_{i d}$ are controller gains.

\section{Identification of the IDC}

Because of the IDC effects among independent joint subsystems, it will reduce the tracking performance via force/position optimal control law. Furthermore, the magnitudes of IDC is totally different with system dynamics $f_{i}\left(x_{i 1}, x_{i 2}\right), g_{i}\left(x_{i 1}\right)$. In this part, an RBF-NN is proposed to approximate the term $h_{i}(x)$.

Using RBF-NN, one can obtain that

$h_{i}=\omega_{i}^{T} \varphi_{i}\left(x_{i}\right)+\varepsilon_{i}$,

where $\varphi_{i}=\exp \left(\frac{\left\|x_{i}-c_{i j}\right\|^{2}}{2 b_{i j}{ }^{2}}\right)$ denotes Gaussian basis function and $c_{i j}, b_{i j}$ are the radial basis function neural network centers and widths. $\omega_{i}$ is ideal neural network weights, $\varepsilon_{i}$ is neural network approximation errors. Define $\hat{\omega}_{i}$ as estimations of $\omega_{i}, \hat{h}_{i}$ is estimation value of $h_{i}(x)$, which can be expressed as:

$\hat{h}_{i}=\hat{\omega}_{i}^{T} \varphi_{i}\left(x_{i}\right)$.

Define estimation errors as $\tilde{\omega}_{i}=\hat{\omega}_{i}-\omega_{i}$. Therefore, we have

$h_{i}-\hat{h}_{i}=\omega_{i}^{T} \varphi_{i}+\varepsilon_{i}-\hat{\omega}_{i}^{T} \varphi_{i}=-\tilde{\omega}_{i}^{T} \varphi_{i}+\varepsilon_{i}$.

The updated law can be defined as follows:

$\dot{\hat{\omega}}_{i}=\gamma_{i} e_{i} \varphi_{i}\left(x_{i}\right)$.
The RBF-NN-based control law is given as:

$u_{i 2}=-\lambda_{i} \dot{e}_{i}-\hat{h}_{i}+\ddot{x}_{i d}-\eta_{i} \operatorname{sgn}\left(e_{i}\right)$.

Theorem 2 Considering the IDC $h_{i}(x)$ in (4) and the dynamic system regarding of the IDC is formulated. The RBF$N N$ presents in (35), and the control law denotes in (38), such that can ensure the IDC term to be asymptotically identified, $\lim _{t \rightarrow \infty} \tilde{h}_{i}(t)=0$.

Proof Choose the Lyapunov function as

$v_{i}=\frac{1}{2} e_{i}^{2}+\frac{1}{2 \gamma_{i}} \tilde{\omega}_{i}^{T} \tilde{\omega}_{i}$

where $\gamma_{i}$ denotes a positive constant.

To apply Lyapunov theorem, the time derivative of $v_{i}$ is obtained as

$$
\begin{aligned}
\dot{v}_{i}= & e_{i}\left(h_{i}-\hat{h}_{i}-\eta_{i} \operatorname{sgn}\left(e_{i}\right)\right)+\frac{1}{\gamma_{i}} \tilde{\omega}_{i}^{T} \dot{\hat{\omega}}_{i} \\
= & e_{i}\left(-\tilde{\omega}_{i}^{T} \varphi_{i}(x)+\varepsilon_{i}-\eta_{i} \operatorname{sgn}\left(e_{i}\right)\right)+\frac{1}{\gamma_{i}} \tilde{\omega}_{i}^{T} \dot{\hat{\omega}}_{i} \\
= & e_{i} \varepsilon_{i}-\eta_{i}\left|e_{i}\right|+\tilde{\omega}_{i}^{T}\left(\frac{1}{\gamma_{i}} \dot{\hat{\omega}}_{i}-e_{i} \varphi_{i}\right) .
\end{aligned}
$$

Then, we can get

$\dot{v}_{i}=\varepsilon_{i} e_{i}-\eta_{i}\left|e_{i}\right| \leq 0$,

where $\eta_{i}>\left|\varepsilon_{i}\right|_{\max }$. This completes the Theorem 2 .

\section{Critic NN implementation}

For finding the optimal control of MRM system (2), one need to solve (13) for the optimal performance index function $\nabla J_{i}^{*}$ and then obtain optimal control $u_{i}^{*}$. However, the HJB equation (13) is a nonlinear partial differential equation and hard to solve. Therefore, we can apply critic NN to approximate $J_{i}^{*}$.

Using critic NN, we have

$J_{i}\left(e_{i}\right)=w_{i F}^{T} \sigma_{i F}\left(e_{i}\right)+\varepsilon_{i F}$,

where $w_{i F} \in R^{N_{i}}$ denotes desired weight, $N_{i}$ means amount of neurons in hidden-layer, $\sigma_{i F}\left(e_{i}\right)$ is activation function, $\varepsilon_{i F}$ means critic NN approximation error. The partial derivative of $J_{i}\left(e_{i}\right)$ is: 
$\nabla J_{i}\left(e_{i}\right)=\left(\nabla \sigma_{i F}\left(e_{i}\right)\right)^{T} w_{i F}+\nabla \varepsilon_{i F}$,

where $\nabla \sigma_{i F}\left(e_{i}\right)=\frac{\partial \sigma_{i F}\left(e_{i}\right)}{\partial e_{i}} \in R^{N_{i} \times n}$ is partial derivative of activation function, $\nabla \varepsilon_{i F}$ is approximate error.

Combining (4), (8), (43), one obtain

$$
\begin{aligned}
0= & \beta_{i} T_{d M}^{2}+N_{i}\left(e_{i}, u_{i}\right)+\Upsilon_{i} \\
& \left(\left(\nabla \sigma_{i F}\left(e_{i}\right)\right)^{T} w_{i F}+\nabla \varepsilon_{i F}\right)^{T}\left(\left(\nabla \sigma_{F}\left(e_{i}\right)\right)^{T} w_{i F}+\nabla \varepsilon_{i F}\right) \\
& +\left(\left(\nabla \sigma_{i F}\left(e_{i}\right)\right)^{T} w_{i F}+\nabla \varepsilon_{i F}\right)^{T} \dot{e}_{i} .
\end{aligned}
$$

Therefore, the Hamiltonian function is:

$$
\begin{aligned}
& H_{i}\left(e_{i}, u_{i}, w_{i F}\right)=\beta_{i} T_{d M}^{2}+N_{i}\left(e_{i}, u_{i}\right) \\
& \quad+\Upsilon_{i} \times\left(\left(\nabla \sigma_{i F}\left(e_{i}\right)\right)^{T} w_{i F}+\nabla \varepsilon_{i F}\right)^{T} \\
& \quad \times\left(\left(\nabla \sigma_{i F}\left(e_{i}\right)\right)^{T} w_{i F}\right. \\
& \left.\quad+\nabla \varepsilon_{i F}\right)+\left(\left(\nabla \sigma_{i F}\left(e_{i}\right)\right)^{T} w_{i F}+\nabla \varepsilon_{i F}\right)^{T} \dot{e}_{i}=e_{i J h},
\end{aligned}
$$

where $e_{i J h}$ means residual error that is caused of $\mathrm{NN}$ approximation. Because $w_{i F}$ is unknown, $J_{i}\left(e_{i}\right)$ can be approximated by critic NN:

$\hat{J}_{i}\left(e_{i}\right)=\hat{w}_{i F}^{T} \sigma_{i F}\left(e_{i}\right)$,

where $\hat{J}_{i}\left(e_{i}\right)$ is estimation of $J_{i}\left(e_{i}\right), \hat{w}_{i F}$ denotes estimation of $w_{i F}$. Then, partial derivative of $\hat{J}_{i}\left(e_{i}\right)$ is:

$\nabla \hat{J}_{i}\left(e_{i}\right)=\left(\nabla \sigma_{i F}\left(e_{i}\right)\right)^{T} \hat{w}_{i F}$

Therefore, HJB function can be written as follows:

$$
\begin{aligned}
H_{i}\left(e_{i}, u_{i}, \hat{w}_{i F}\right)= & \beta_{i} T_{d M}^{2}+\Upsilon_{i}\left(\left(\nabla \sigma_{i F}\left(e_{i}\right)\right)^{T} \hat{w}_{i F}\right)^{T} \\
& \left(\left(\nabla \sigma_{i F}\left(e_{i}\right)\right)^{T} \hat{w}_{i F}\right)+\left(\left(\nabla \sigma_{i F}\left(e_{i}\right)\right)^{T} \hat{w}_{i F}\right) \dot{e}_{i} \\
& +N_{i}\left(e_{i}, u_{i}\right)=e_{i J} .
\end{aligned}
$$

Define objective function $E_{i J}=\frac{1}{2} e_{i J}^{T} e_{i J}$ which is minimized by gradient decent algorithm for adjusting $\hat{w}_{i F}$, which is updated by

$\dot{\hat{w}}_{i F}=-\alpha_{i F} e_{i J} \nabla \sigma_{i F}\left(e_{i}\right) \dot{e}_{i}$,

where $\alpha_{i F}$ means updated rate of critic NN. Denote $v_{i}=$ $\nabla \sigma_{i F}\left(e_{i}\right) \dot{e}_{i}$, and consider there exists a positive constant $v_{i L}$ that $\left\|v_{i}\right\| \leq v_{i L}$.

Define weight approximation error as

$\tilde{w}_{i F}=w_{i F}-\hat{w}_{i F}$.
By (45), (48) and (49), we have

$e_{i J}=e_{i J h}-\tilde{w}_{i F}^{T} v_{i}$

The weight approximation error is

$$
\dot{\tilde{w}}_{i F}=-\dot{\hat{w}}_{i F}=\alpha_{i F} e_{i J} v_{i}=\alpha_{i F}\left(e_{i J h}-\tilde{w}_{i F}^{T} v_{i}\right) v_{i}
$$

Combining (12) and (43), the desired control policy is as follows:

$u_{i 3}{ }^{*}=-\frac{1}{2} k_{q w e i} R_{i}^{-1} g_{i}^{T}\left(\left(\nabla \sigma_{i F}\left(e_{i}\right)\right)^{T} w_{i F}+\nabla \varepsilon_{i F}\right)$.

Therefore, the approximated force/position optimal control policy $\hat{u}_{i 3}^{*}$ is

$\hat{u}_{i 3}^{*}=-\frac{1}{2} k_{q w e i} R_{i}^{-1} \hat{g}_{i}^{T}\left(\nabla \sigma_{i F}\left(e_{i}\right)\right)^{T} \hat{w}_{i F}$

By combining (33), (38) with (54), the proposed decentralized force/position approximate optimal control law $u_{i}^{*}$ is

$$
\begin{aligned}
\hat{u}_{i}^{*}= & k_{i p}\left(x_{i d}-\hat{x}_{i 1}\right)-k_{i d} \hat{x}_{i 2}-\hat{x}_{i 3}-\lambda_{i} \dot{e}_{i}-\hat{h}_{i}+\ddot{x}_{i d} \\
& -\eta_{i} \operatorname{sgn}\left(e_{i}\right)-\frac{1}{2} k_{q w e i} R_{i}^{-1} \hat{g}_{i}^{T}\left(\nabla \sigma_{i F}\left(e_{i}\right)\right)^{T} \hat{w}_{i F} .
\end{aligned}
$$

\section{Policy iteration algorithm}

To address HJB equation, PI algorithm is shown as follows.

Step 1: Let $k=0$, start with an initial admissible control $u_{i}{ }^{(0)}$, and select a small positive constant $\varepsilon_{i}$.

Step 2: Let $k>0$, based on $u_{i}^{(k)}$, solve $\hat{J}_{i}^{(k)}\left(e_{i}\right)$ via $0=$ $\beta_{i} T_{d M}^{2}+N_{i}\left(e_{i}, u_{i}^{(k)}\right)+\Upsilon_{i}\left(\nabla \hat{J}_{i}^{(k+1)}\left(e_{i}\right)\right)^{2}+\nabla \hat{J}_{i}^{(k+1)}\left(e_{i}\right)^{T}$. $\left(k_{\text {qwei }}\left(f_{i}\left(\hat{x}_{i 1}, \hat{x}_{i 2}\right)+\hat{g}_{i}\left(\hat{x}_{i 1}\right)\left(u_{i 1}+u_{i 2}+u_{i 3}^{*}+\tau_{c i}\right)\right.\right.$ $\left.\left.+\hat{h}_{i}(x)+d_{i}\right)+v_{i}\right)$, with $\hat{J}^{(k+1)}(0)=0$.

Step 3: Update $u_{i}{ }^{(k)}$ through $u_{i}{ }^{(k+1)}$ $=-\frac{1}{2} k_{q w e i} R_{i}^{-1} \hat{g}_{i}^{T}\left(\hat{x}_{i 1}\right) \nabla \hat{J}_{i}^{(k+1)}\left(e_{i}\right)$.

Step 4: While $\left\|\hat{J}_{i}^{(k+1)}\left(e_{i}\right)-\hat{J}_{i}^{(k)}\left(e_{i}\right)\right\| \leq \varepsilon_{i}$, stop and get optimal control; else, let $k=k+1$ and back to Step 2 .

The algorithm will converge to optimal performance index function and control policy, $\hat{J}_{i}^{(k)}\left(e_{i}\right) \rightarrow \hat{J}_{i}^{*}\left(e_{i}\right), u_{i}{ }^{(k)} \rightarrow u_{i}{ }^{*}$ while $t \rightarrow \infty$.

\section{Stability analysis}

We investigate stability of closed-loop MRM system under the force/position optimal control law (55), which is:

Theorem 3 Considering an n-DOF MRM system, with the dynamic model formulation (2) and the IDC existed in (4). 
The approximated force/position optimal tracking control policy (55) with ESO (15) and RBF-NN (35) can ensure the asymptotic stability of the closed-loop manipulator system with approximate (46) that is updated by (49).

Proof Choose the Lyapunov candidate function as:

$$
\begin{aligned}
V(t) & =\sum_{i=1}^{n} V_{i}(t)=\sum_{i=1}^{n}\left(\frac{1}{2} e_{i}^{T} e_{i}+J_{i}{ }^{*}\left(e_{i}\right)\right. \\
& \left.+\frac{1}{2} \int_{t}^{\infty}\left(\tau_{c i}^{2}+T_{d M}^{2}\right) d t+\frac{1}{2 \alpha_{i F}} \tilde{w}_{i F}^{T} \tilde{w}_{i F}\right) .
\end{aligned}
$$

The time derivative of $V(t)$ is:

$$
\begin{aligned}
\dot{V}(t) & =\sum_{i=1}^{n}\left(e_{i}^{T} \dot{e}_{i}+\left(\nabla J_{i}^{*}\left(e_{i}\right)\right)^{T} \dot{e}_{i}-\frac{1}{2}\left\|\tau_{c i}\right\|^{2}\right. \\
& \left.-\frac{1}{2}\left\|T_{d M}\right\|^{2}+\frac{1}{\alpha_{i F}} \tilde{w}_{i F}^{T} \dot{\tilde{w}}_{i F}\right) .
\end{aligned}
$$

According to (8) and (13), we have

$\dot{V}(t)=\sum_{i=1}^{n}\left(\begin{array}{c}e_{i}^{T} \cdot\left(k_{q w e i}\left(\begin{array}{c}f_{i}\left(\hat{x}_{i 1}, \hat{x}_{i 2}\right)+\hat{g}_{i}\left(\hat{x}_{i 1}\right)\left(u_{i 1}+\right. \\ \left.u_{i 2}+u_{i 3}{ }^{*}+\tau_{\mathrm{ci}}\right)+\hat{h}_{i}(x)+d_{i}\end{array}\right)+v_{i}\right) \\ -\beta_{i} T_{d M}^{2}-e_{i}^{T} Q_{i} e_{i}-u_{i}^{T} R_{i} u_{i}-\Upsilon_{i}\left(\nabla J_{i}{ }^{*}\left(e_{i}\right)\right)^{2} \\ -\frac{1}{2}\left\|\tau_{c i}\right\|^{2}-\frac{1}{2}\left\|T_{d M}\right\|^{2}+\tilde{w}_{i F}^{T} e_{i J h} v_{i}-\left\|\tilde{w}_{i F}^{T} v_{i}\right\|^{2}\end{array}\right)$.

Because of $f_{i}\left(\hat{x}_{i 1}, \hat{x}_{i 2}\right)$ is locally Lipchitz, assuming $L_{i f}>0$ is a positive constant and $\left\|f_{i}\left(\hat{x}_{i 1}, \hat{x}_{i 2}\right)\right\| \leq L_{i f}\left\|e_{i}\right\|$, $\left\|\hat{g}\left(\hat{x}_{i 1}\right)\right\| \leq \omega_{i g}$ with $\omega_{i g}>0$. Assume $\left\|\ddot{q}_{i d}\right\| \leq \delta_{i}$, $\left\|\dot{e}_{q i}\right\| \leq \xi_{i},\left\|e_{\tau i}\right\| \leq \eta_{i}$, by Young's inequality, we have

$$
\dot{V}(t) \leq \sum_{i=1}^{n}\left(\begin{array}{c}
\left\|e_{i}\right\|\left(k_{q w e i}\left(\omega_{i g}\left(u_{i}+\tau_{c i}+T_{d M}\right)+L_{i f}\left\|e_{i}\right\|\right)+v_{i}\right) \\
-\beta_{i} T_{d M}^{2}-e_{i}^{T} Q_{i} e_{i}-u_{i}^{T} R_{i} u_{i}-\Upsilon_{i}\left(\nabla J_{i}{ }^{*}\left(e_{i}\right)\right)^{2} \\
-\frac{1}{2}\left\|\tau_{c i}\right\|^{2}-\frac{1}{2}\left\|T_{d M}\right\|^{2}+\tilde{w}_{i F}^{T} e_{i J h} v_{i}-\left\|\tilde{w}_{i F}^{T} v_{i}\right\|^{2}
\end{array}\right)
$$

$\dot{V}(t) \leq \sum_{i=1}^{n}\left(\begin{array}{l}-\left\|e_{i}\right\|\left(\Psi_{i 1}\left\|e_{i}\right\|+k_{q w e i} \delta_{i}-k_{w e i} \xi_{i}-k_{\tau i} \eta_{i}\right) \\ -\Psi_{i 2}\left\|u_{i}\right\|^{2}-\Upsilon_{i}\left(\nabla J_{i}^{*}\left(e_{i}\right)\right)^{2}-\frac{1}{2}\left(\left\|\tilde{w}_{i F}^{T} v_{i}\right\|^{2}-\left\|e_{i J h}\right\|^{2}\right)-\beta_{i} T_{d M}^{2}\end{array}\right)$.

Define $\Psi_{i 1}=\lambda_{\min }\left(Q_{i}\right)-\frac{3}{2} k_{q w e i}^{2} \omega_{i g}^{2}-k_{q w e i} L_{i f}, \Psi_{i 2}=$ $\lambda_{\min }\left(R_{i}\right)-\frac{1}{2}$. Then

Fig. 1 MRMs with different configurations under uncertain environmental constraints

$=\sum_{i=1}^{n}\left(\begin{array}{l}v_{i}\left\|e_{i}\right\|+k_{q w e i} L_{i f}\left\|e_{i}\right\|^{2}+k_{q w e i} \omega_{i g}\left\|e_{i}\right\|\left\|u_{i}\right\|+ \\ k_{q w e i} \omega_{i g}\left\|e_{i}\right\|\left\|\tau_{c i}\right\|+k_{q w e i} \omega_{i g}\left\|e_{i}\right\|\left\|T_{d M}\right\|-\beta_{i} T_{d M}^{2} \\ -e_{i}{ }^{T} Q_{i} e_{i}-u_{i}{ }^{T} R_{i} u_{i}-\Upsilon_{i}\left(\nabla J_{i}{ }^{*}\left(e_{i}\right)\right)^{2}-\frac{1}{2}\left\|\tau_{c i}\right\|^{2} \\ -\frac{1}{2}\left\|T_{d M}\right\|^{2}+\tilde{w}_{i F}^{T} e_{i J h} v_{i}-\left\|\tilde{w}_{i F}^{T} v_{i}\right\|^{2}\end{array}\right)$

$\leq \sum_{i=1}^{n}\left(\begin{array}{l}v_{i}\left\|e_{i}\right\|+k_{q w e i} L_{i f}\left\|e_{i}\right\|^{2}+\frac{3}{2} k_{q w e i}^{2} \omega_{i g}^{2}\left\|e_{i}\right\|^{2}+\frac{1}{2}\left\|u_{i}\right\|^{2} \\ +\frac{1}{2}\left\|\tau_{c i}\right\|^{2}+\frac{1}{2}\left\|T_{d M}\right\|^{2}-\lambda_{\min }\left(Q_{i}\right)\left\|e_{i}\right\|^{2}-\lambda_{\min }\left(R_{i}\right) \\ \left\|u_{i}\right\|^{2}-\Upsilon_{i}\left(\nabla J_{i}{ }^{*}\left(e_{i}\right)\right)^{2}-\frac{1}{2}\left\|\tau_{c i}\right\|^{2}-\frac{1}{2}\left\|T_{d M}\right\|^{2} \\ -\frac{1}{2}\left(\left\|\tilde{w}_{i F}^{T} v_{i}\right\|^{2}-\left\|e_{i J h}\right\|^{2}\right)-\beta_{i} T_{d M}^{2}\end{array}\right)$

$\leq \sum_{i=1}^{n}\left(\begin{array}{c}\left\|e_{i}\right\|\left(\begin{array}{c}v_{i}-\lambda_{\min }\left(Q_{i}\right)\left\|e_{i}\right\|+k_{q w e i} \\ L_{i f}\left\|e_{i}\right\|+\frac{3}{2} k_{q w e i}^{2} \omega_{i g}^{2}\left\|e_{i}\right\|\end{array}\right)-\Upsilon_{i}\left(\nabla J_{i}{ }^{*}\left(e_{i}\right)\right)^{2}- \\ \left(\lambda_{\min }\left(R_{i}\right)-\frac{1}{2}\right)\left\|u_{i}\right\|^{2}-\frac{1}{2}\left(\left\|\tilde{w}_{i F}^{T} v_{i}\right\|^{2}-\left\|e_{i J h}\right\|^{2}\right)-\beta_{i} T_{d M}^{2}\end{array}\right)$

$\leq \sum_{i=1}^{n}\left(\begin{array}{l}-\left\|e_{i}\right\|\left(\left\|e_{i}\right\|\left(\lambda_{\min }\left(Q_{i}\right)-\frac{3}{2} k_{q w e i}^{2} \omega_{i g}^{2}-k_{q w e i} L_{i f}\right)-v_{i}\right) \\ -\Upsilon_{i}\left(\nabla J_{i}{ }^{*}\left(e_{i}\right)\right)^{2}-\left(\lambda_{\min }\left(R_{i}\right)-\frac{1}{2}\right)\left\|u_{i}\right\|^{2}- \\ \frac{1}{2}\left(\left\|\tilde{w}_{i F}^{T} v_{i}\right\|^{2}-\left\|e_{i J h}\right\|^{2}\right)-\beta_{i} T_{d M}^{2}\end{array}\right)$.

So that, we have $\dot{V}(t) \leq 0$ while $e_{i}$ lies outside the compact set $\Omega_{i 1}=\left\{e_{i}:\left\|e_{i}\right\| \leq \frac{-k_{q w e i} \delta_{i}+k_{w e i} \xi_{i}+k_{\tau i} \eta_{i}}{\Psi_{i 1}}\right\}$, and $\Omega_{i 2}=\left\{\tilde{w}_{i F}\left\|\tilde{w}_{i F}\right\| \leq\left\|\frac{e_{i J h}}{v_{i L}}\right\|\right\}$, if following 
Table 1 Control parameter setting

\begin{tabular}{llllllllllll}
\hline Name & Value & Name & Value & Name & Value & Name & Value & Name & Value & Name & Value \\
\hline$k_{i p}$ & 1.5 & $k_{i d}$ & 0.3 & $\lambda_{i}$ & 0.5 & $\eta_{i}$ & 0.8 & $\alpha_{i F}$ & 0.9 & $k_{q w e i}$ & 1 \\
$\beta_{i}$ & 1 & $\beta_{i 01}$ & 2.33 & $\beta_{i 02}$ & 1.56 & $\beta_{i 03}$ & 0.3 & $T_{d M}$ & 2 & $\Upsilon_{i}$ & 1 \\
\hline
\end{tabular}

conditions hold

$$
\left\{\begin{array}{c}
\lambda_{\min }\left(Q_{i}\right) \geq k_{q w e i} L_{i f}+\frac{3}{2} k_{q w e i}^{2} \omega_{i g}^{2} \\
\lambda_{\min }\left(R_{i}\right) \geq \frac{1}{2}
\end{array}\right.
$$

Moreover, (60) means that $\dot{V}(t) \leq 0$ for $e_{i} \neq 0$ while only (61) is satisfied. Hence, decentralized force/position approximate optimal controllers asymptotically stable.

\section{Simulation}

\section{Simulation setup}

By referring Newton-Euler iteration method and reference [36], two MRMs (see Fig. 1) are given for simulation. The end-effector is operating along with the constrained plane (the constrained equation). According to the constrained equation, we can obtain the desired trajectory and the MRM system is under uncertain environment such as contact or collision. The dynamic model for configuration $\mathrm{A}$ is:

$$
\begin{gathered}
M(q)=\left[\begin{array}{ll}
0.6066+0.36 \cos \left(q_{2}\right) & 0.1233+0.18 \cos \left(q_{2}\right) \\
0.1233+0.18 \cos \left(q_{2}\right) & 0.1233
\end{array}\right] \\
C(q, \dot{q})=\left[\begin{array}{cc}
-0.36 \sin \left(q_{2}\right) \dot{q}_{2} & -0.18 \dot{q}_{2} \sin \left(q_{2}\right) \\
0.18 \sin \left(q_{2}\right)\left(\dot{q}_{1}-\dot{q}_{2}\right) & 0.18 \dot{q}_{1} \sin \left(q_{2}\right)
\end{array}\right] \\
G(q)=\left[\begin{array}{c}
-5.88 \sin \left(q_{1}+q_{2}\right)-17.64 \sin \left(q_{1}\right) \\
-5.88 \sin \left(q_{1}+q_{2}\right)
\end{array}\right],
\end{gathered}
$$

and configuration $\mathrm{B}$ is:

$$
\begin{aligned}
& M(q)=\left[\begin{array}{cc}
0.17-0.1166 \cos ^{2}\left(q_{2}\right) & -0.06 \cos \left(q_{2}\right) \\
-0.06 \cos \left(q_{2}\right) & 0.1233
\end{array}\right] \\
& C(q, \dot{q})=\left[\begin{array}{cc}
-0.1166 \dot{q}_{2} \sin \left(2 q_{2}\right) & -0.06 \sin \left(q_{2}\right) \dot{q}_{2} \\
\left(0.06 \sin \left(q_{2}\right) \dot{q}_{2}-0.0583 \sin \left(2 q_{2}\right) \dot{q}_{1}\right) & -0.06 \dot{q}_{1} \sin \left(q_{2}\right)
\end{array}\right]
\end{aligned}
$$

and for configuration $\mathrm{B}$ can be described as

$$
G(q)=\left[\begin{array}{c}
0 \\
-5.88 \sin \left(q_{1}+q_{2}\right)
\end{array}\right] \text {. }
$$

For configuration $\mathrm{A}$, the constrained equation for MRM is: $\Phi(q)=-1.5+l_{2} \cos q_{2}+l_{1}=0$ and for configuration $\mathrm{B}$ is $\Phi(q)=-1+l_{2} \cos q_{2}+l_{1} \cos q_{1}=0$, where $l_{1}, l_{2}$ are the lengths of two manipulator links. The desired trajectory as well as desired contact force for configuration A are:

$$
\begin{aligned}
& q_{1 d}=\sin (2 t)+0.2 \cos (t), q_{2 d}=\frac{\pi}{3}, f_{d}=10, \\
& q_{1 d}=0.5 \sin (2 t)+\cos (t), \\
& q_{2 d}=\arccos \left(\frac{1-l_{1} \cos (\cos (t)+0.5 \sin (2 t))}{l_{2}}\right), f_{d}=10 .
\end{aligned}
$$

The parameter definition is: $Q_{i}=R_{i}=I$. The critic NN is selected as 2-3-1, and $\hat{w}_{i F}=\left[\hat{w}_{i F 1}, \hat{w}_{i F 2}, \hat{w}_{i F 3}\right]^{T}$ with the initial values $\hat{W}_{i F}=\left[\begin{array}{lll}10 & 15 & 20\end{array}\right]^{T}$. The active function
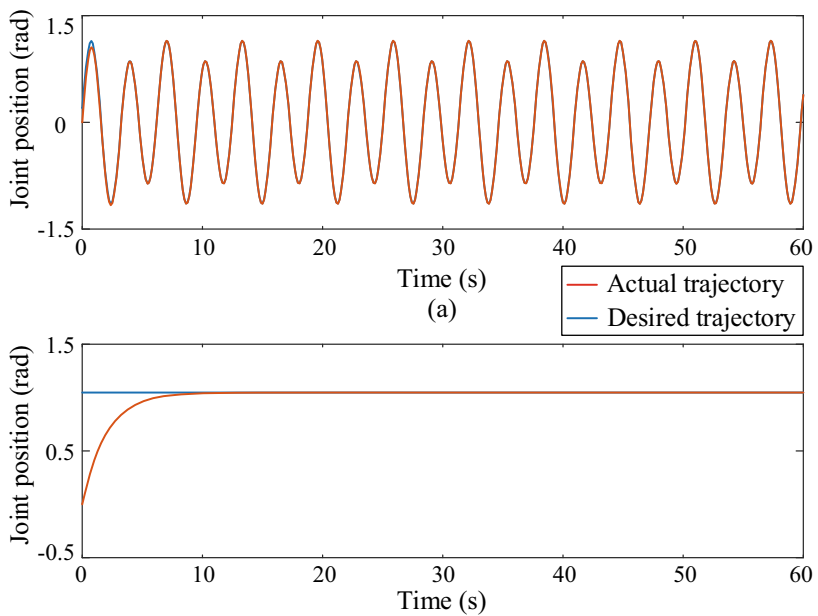

(b)

Fig. 2 Trajectory tracking for configuration A with proposed methods. a Joint one b Joint two

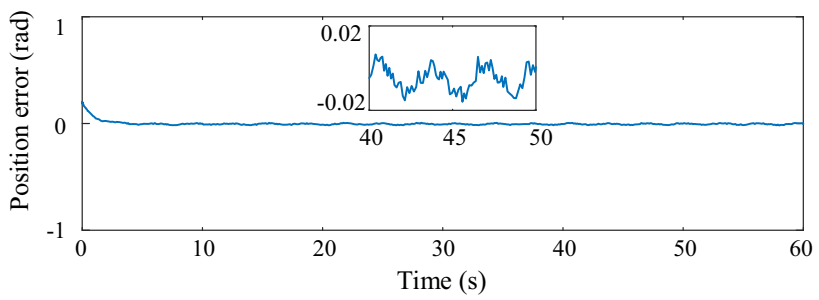

(a)

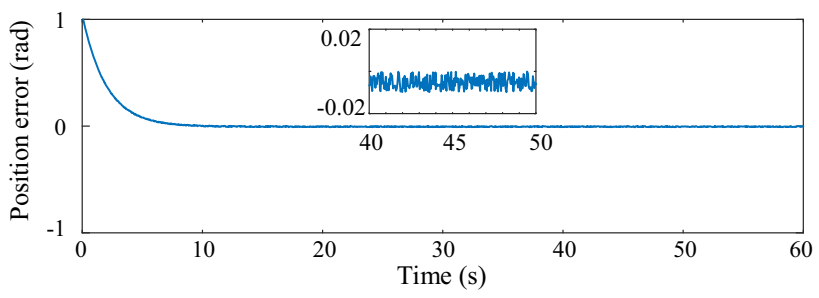

(b)

Fig. 3 Position error for configuration A with existing methods. a Joint one b Joint Two

is selected as $\sigma_{i F}=\left[e_{i}^{2}, e_{i}^{2}, e_{i}^{2}\right]$, and the control parameters are in Table 1.

\section{Simulation results}

(1) Trajectory tracking performance

Figure 2 is joint position curve of configuration A under proposed method. The proposed method is effectiveness because 


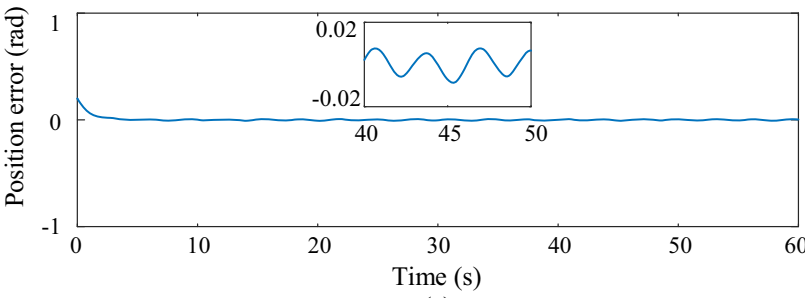

(a)

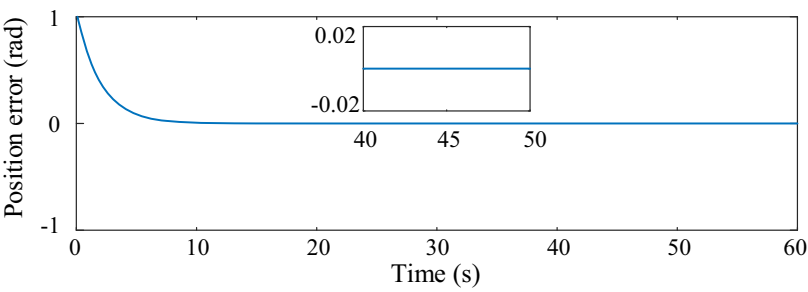

(b)

Fig. 4 Position error for configuration A with proposed methods. a Joint one b Joint two
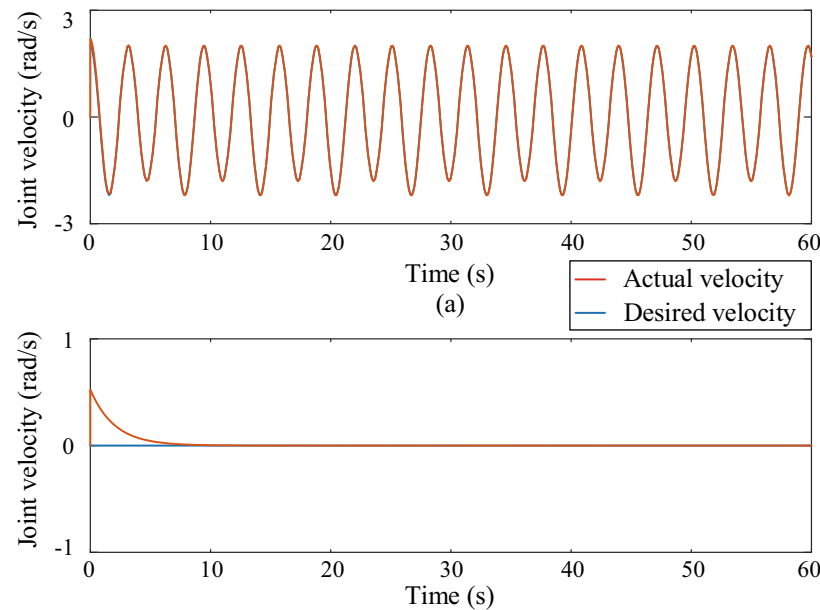

(b)

Fig. 5 Velocity curves for configuration A with proposed methods. a Joint one b Joint Two

the actual position can follow the desired trajectory very fast. Figures 3 and 4 illustrate joint position tracking error curves under existing and proposed method. In Fig. 3, we can observe position errors are obvious at initial times and amplitude of steady-state error is 0.04 rad. From Fig. 4, position errors can follow very fast. Figure 5 is the velocity tracking curves under the proposed method of configuration A. Figures 6 and 7 are velocity error curves under existing method and proposed method. Because of the existing methods have not considered the influence of IDC effects, so that the tracking error is larger than the proposed method.

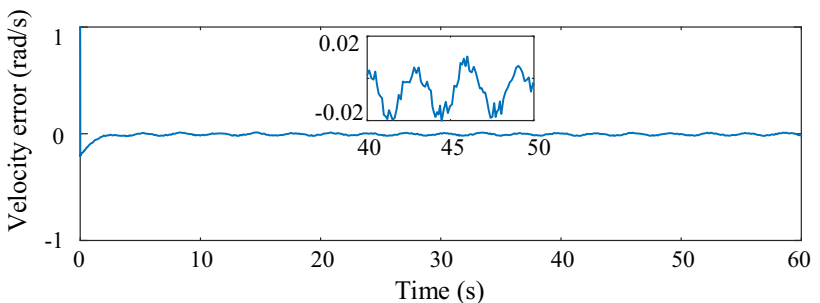

(a)

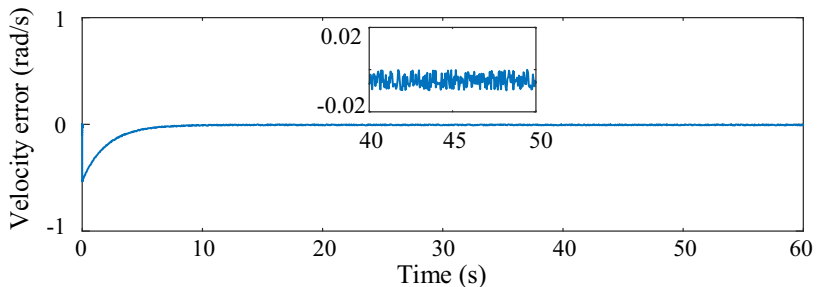

(b)

Fig. 6 Velocity error for configuration A with existing methods. a Joint one b Joint two

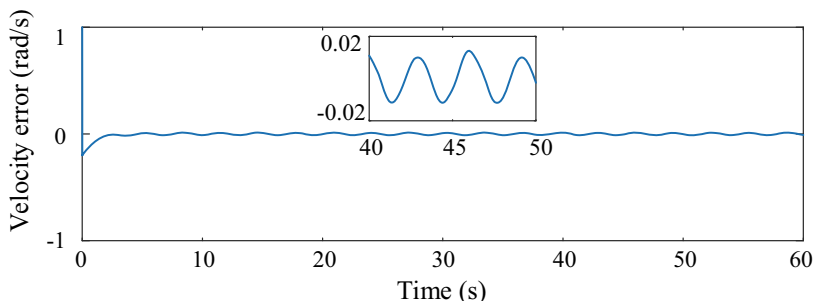

(a)

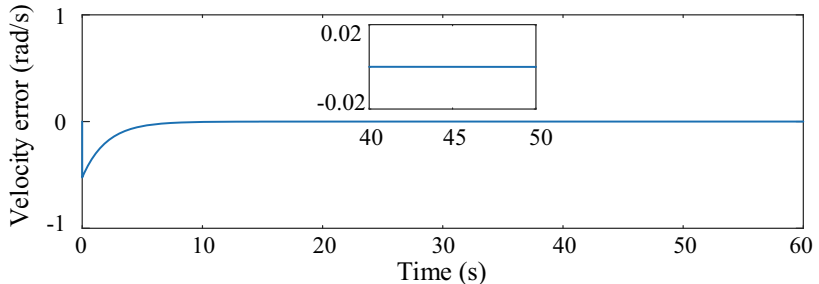

(b)

Fig. 7 Velocity error for configuration A with proposed methods. a Joint one b Joint two

\section{(2) Control torque}

Figure 8 is control torque curve with existing method of configuration A. For joint one, because of adjustment of the weight of critic NN, the initial control torque is large. For joint two, the control torque has a sudden variety in some time. That is because the method without dynamic compensation. Figure 9 is torque curve of configuration A with the proposed method. The output torques have been optimized with an appropriate behavior because of the developed scheme. 


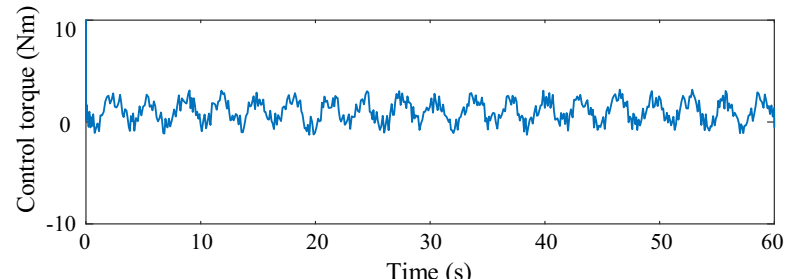

(a)

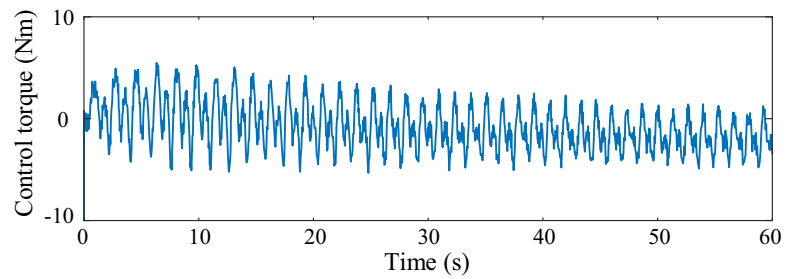

(b)

Fig. 8 Control torque for configuration A with existing methods. a Joint one b Joint two

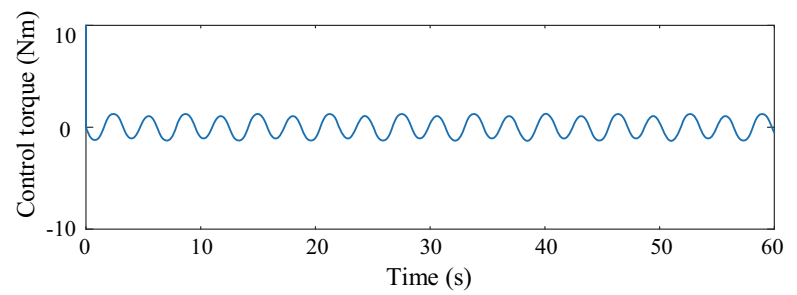

(a)

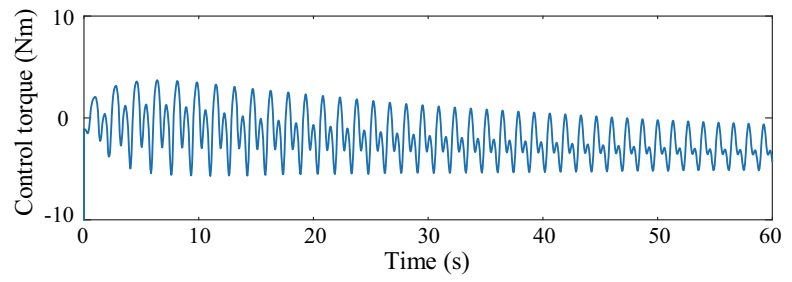

(b)

Fig. 9 Control torque for configuration A with proposed methods. a Joint one b Joint two

\section{(3) Force performance}

Figures 10 and 11 represent the contact force and force error curves under the existence and proposed methods. One can observe that the proposed method can follow the contact force quickly and force error can be a very small value in a very short time which is attributed to the decentralized optimal force/position control method.

(4) Critic NN adjustment

Figure 12 is position error performance with different learning rate for configuration $\mathrm{A}$. It is obvious that different learning rate can achieve different solution. If the parameter is too big, it will lead overtraining for iteration process and

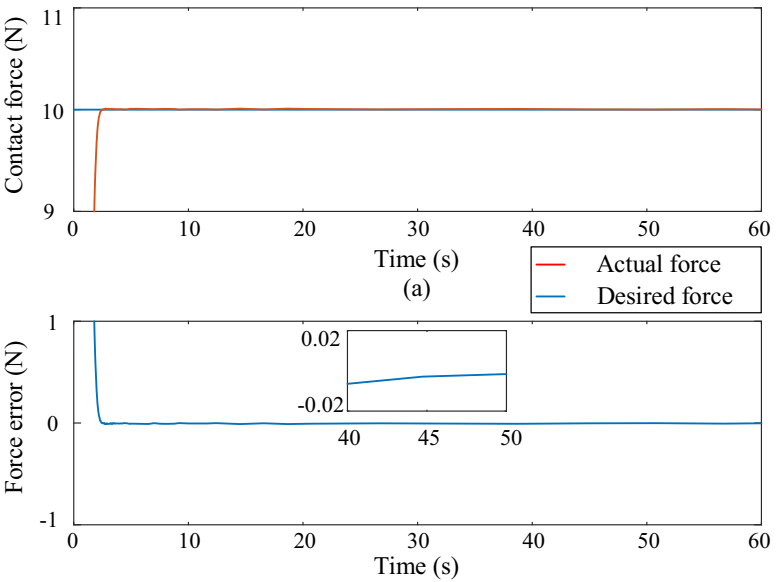

(b)

Fig. 10 Force curve for configuration A with existing methods. a Contact force. b Force error

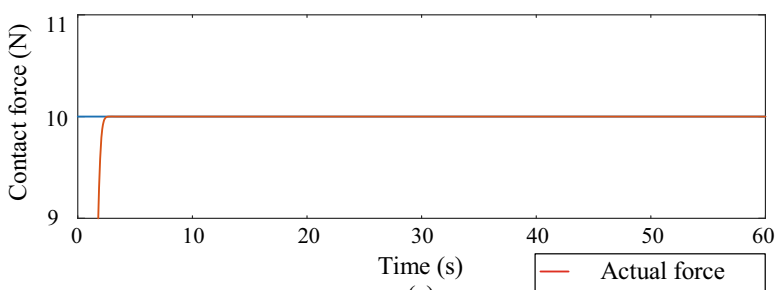

(a) - Desired force

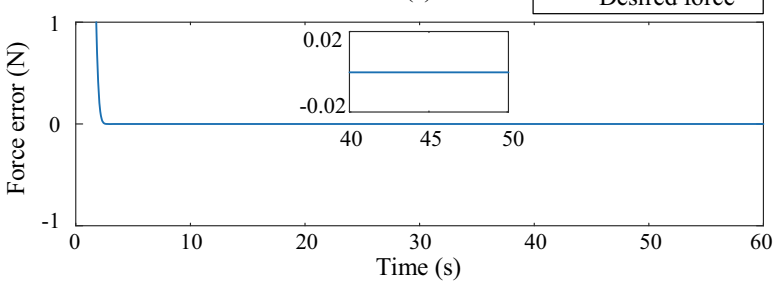

(b)

Fig. 11 Force curve for configuration A with proposed methods. a Contact force. $\mathbf{b}$ Force error

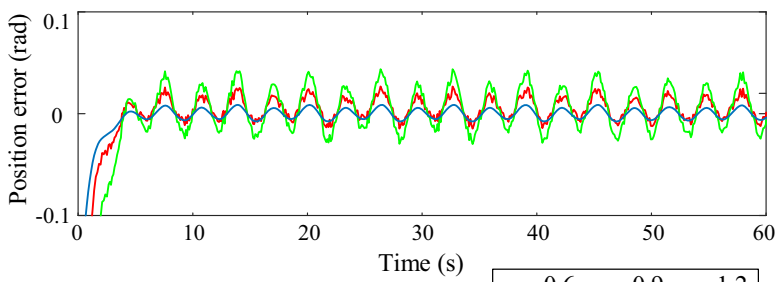

(a) $-0.6-0.9-1.2$

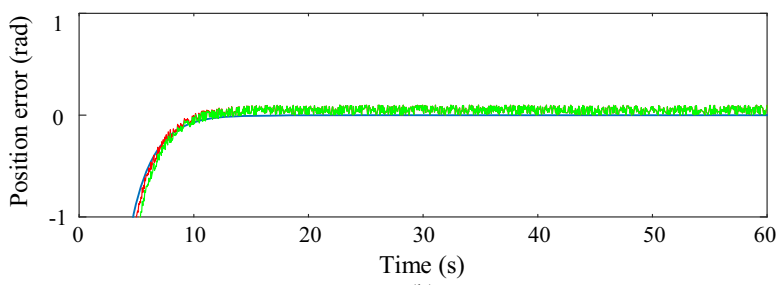

(b)

Fig. 12 Position error performance with different learning rate for configuration A. a Joint one b Joint two 

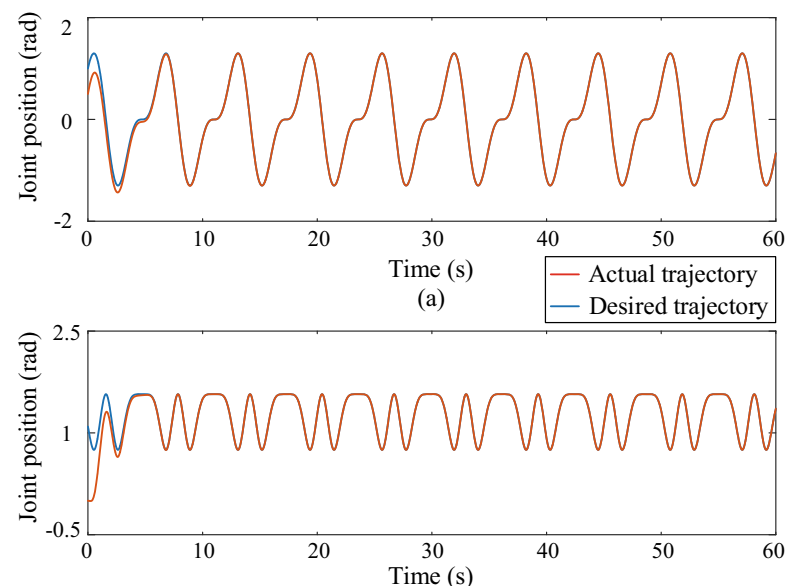

(b)

Fig. 13 Trajectory tracking for configuration B with proposed methods. a Joint one b Joint two

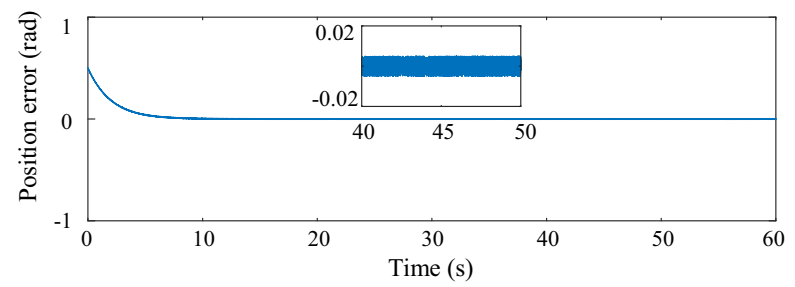

(a)

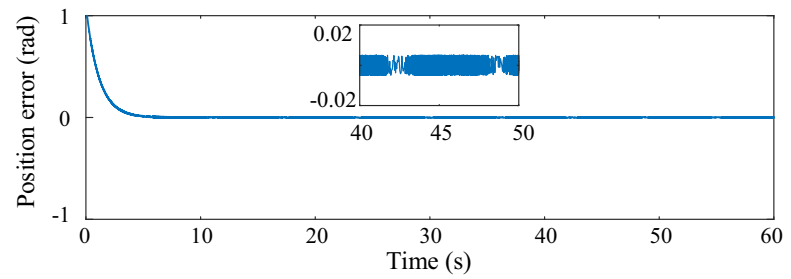

(b)

Fig. 14 Position error for configuration B with existing methods. a Joint one b Joint two

the performance index will not improve. Besides the properties of accuracy in robotic system, fast response is also an important index. If $\alpha_{i F}$ select very small, the accuracy of tracking index will be discounted that means undertraining. Based on different simulation analysis, we select $\alpha_{i F}$ as 0.9 .

Figures 13, 14, 15, 16, 17, 18, 19, 20, 21 and 22 are position tracking curves, position error, velocity curves, velocity error, control torque and contact force, force error curves for configuration B. The results are similar with configuration A. It improves that proposed method without the requirements of adjusting parameters. For the simulations, one can get the decentralized optimal force/position method can guarantee stability and accuracy.

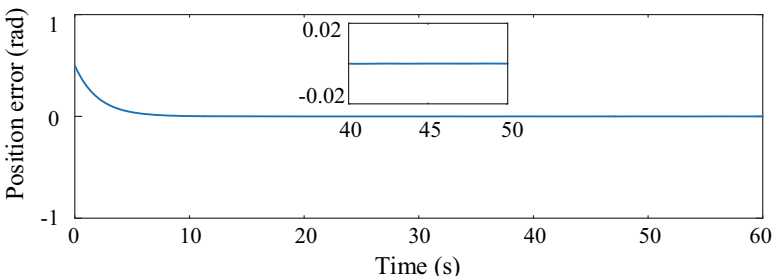

(a)

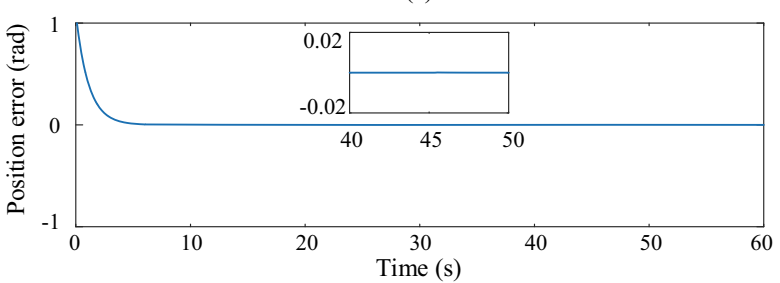

(b)

Fig. 15 Position error for configuration B with proposed methods. a Joint one b Joint two
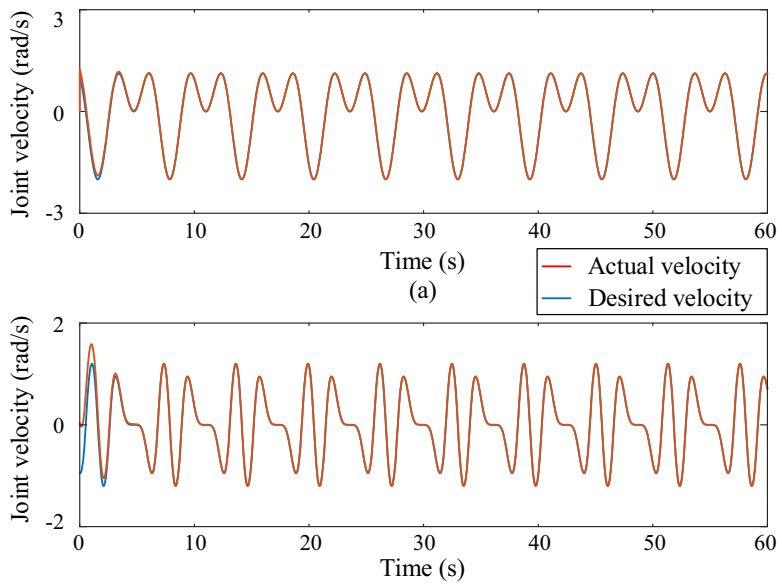

(b)

Fig. 16 Velocity curves for configuration B with proposed methods. a Joint one b Joint two

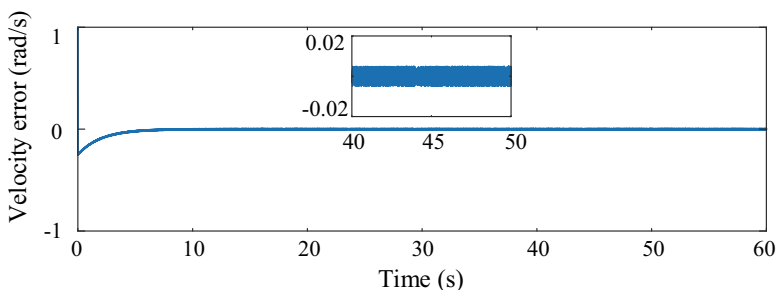

(a)

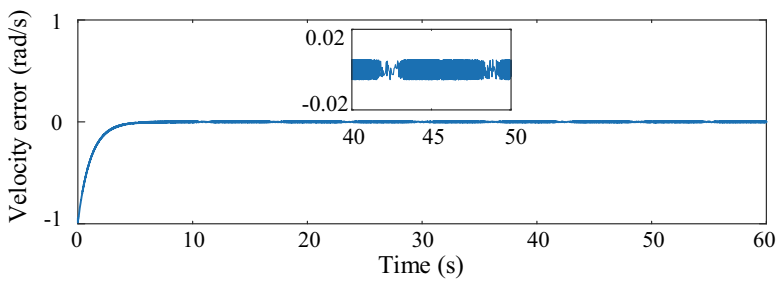

(b)

Fig. 17 Velocity error for configuration B with existing methods. a Joint one. b Joint two 


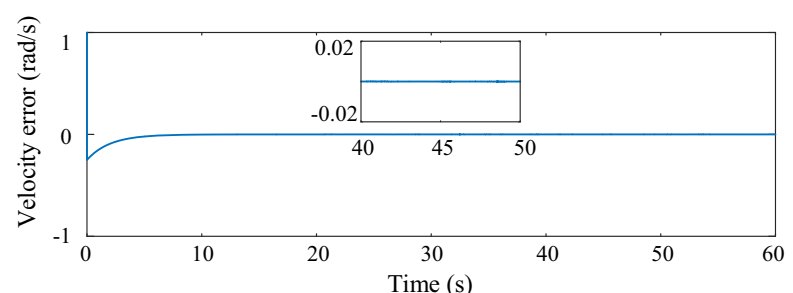

(a)

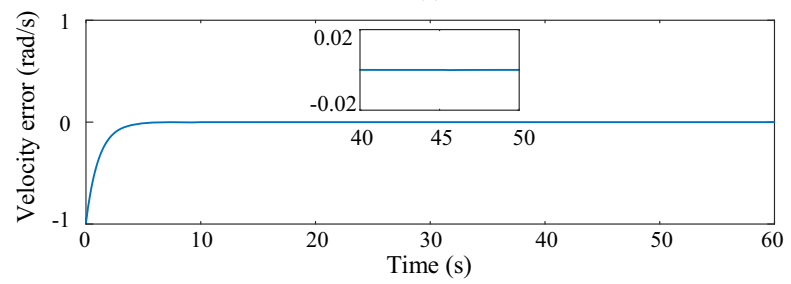

(b)

Fig. 18 Velocity error for configuration B with proposed methods. a Joint one. b Joint two

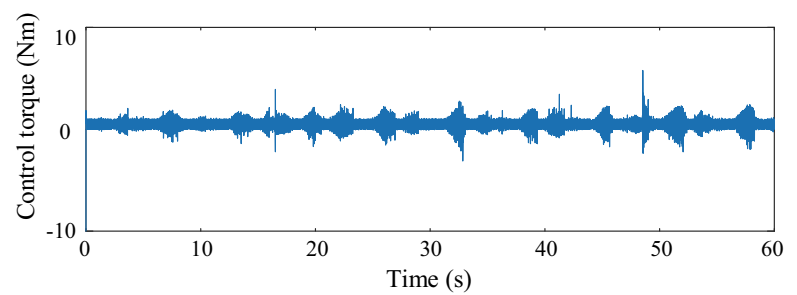

(a)

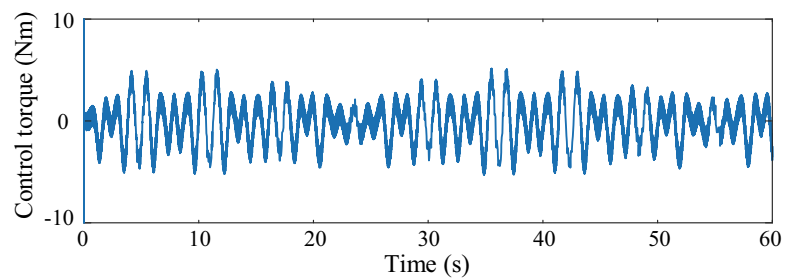

(b)

Fig. 19 Control torque for configuration B with existing methods. a Joint one. b Joint two

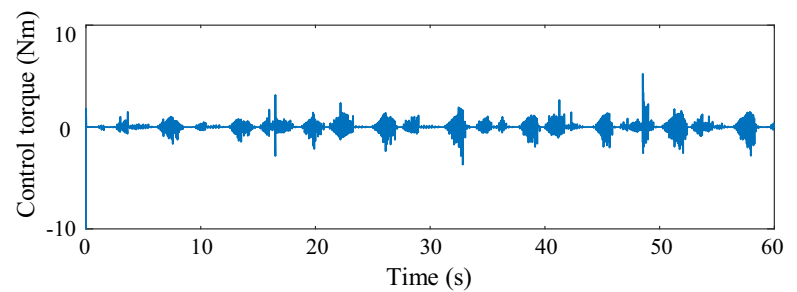

(a)

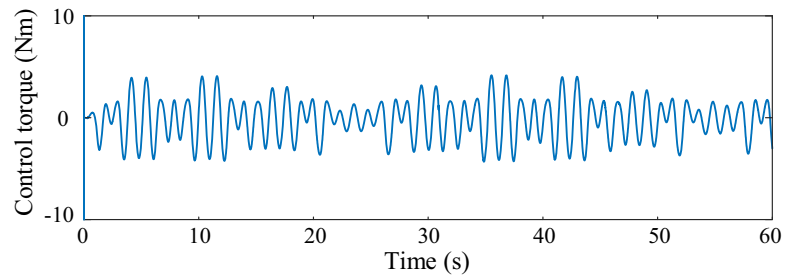

(b)

Fig. 20 Control torque for configuration B with proposed methods. a Joint one. b Joint two

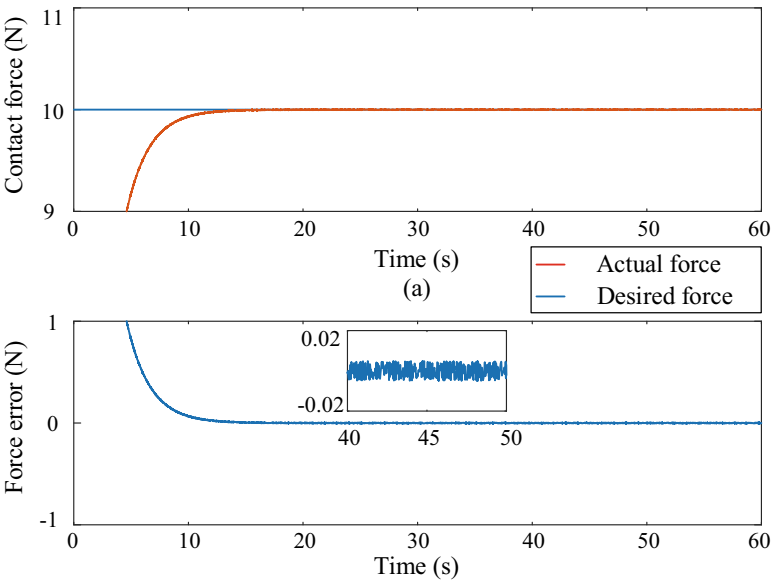

(b)

Fig. 21 Force curve for configuration B with existing methods. a Contact force. b Force error

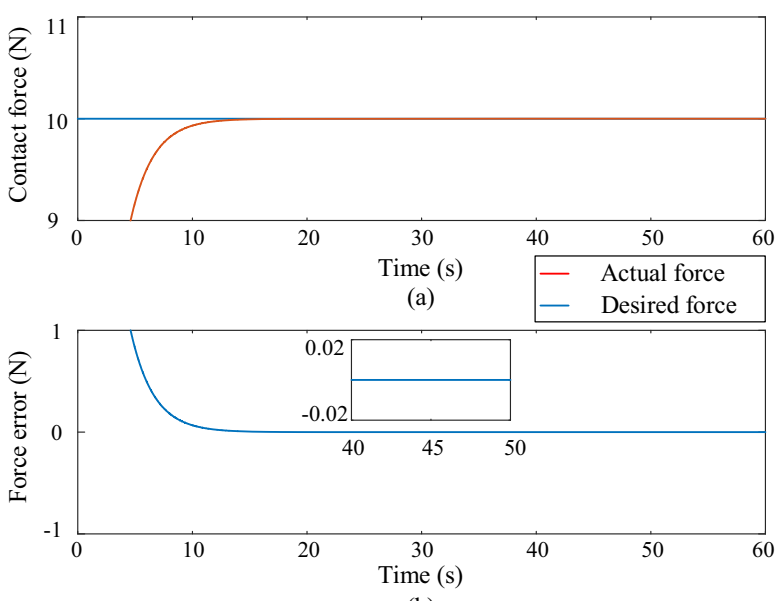

(b)

Fig. 22 Force curve for configuration B with proposed methods. a Contact force. b Force error

\section{Conclusion}

A critic-observer decentralized force/position approximate optimal control method is presented to address the problems of optimal trajectory and force tracking problem for MRMs with uncertain environmental constraints. The dynamic model of the MRM systems is formulated as an integration of joint subsystems and the unknown manipulator dynamics are identified by the developed ESO. Then, an RBF-NN is developed to deal with IDC effects among the independent joint subsystems. Based on ADP and PI algorithms, HJB equation is approximately solved using critic $\mathrm{NN}$ and then the approximated optimal control policy can be derived. The closed-loop manipulator system is proved to be asymptotic stable by using the Lyapunov theory. Simulation results are provided to demonstrate the effectiveness and advantage of the proposed control method finally. 
Acknowledgements This work is supported by the National Natural Science Foundation of China (Grant Nos. 61773075 and 61703055), the Scientific Technological Development Plan Project in Jilin Province of China (Grant No. 20200801056GH) and the Science and Technology project of Jilin Provincial Education Department of China during the 13th 5-Year Plan Period (JJKH20200672KJ, JJKH20200673KJ and JJKH20200674KJ).

Open Access This article is licensed under a Creative Commons Attribution 4.0 International License, which permits use, sharing, adaptation, distribution and reproduction in any medium or format, as long as you give appropriate credit to the original author(s) and the source, provide a link to the Creative Commons licence, and indicate if changes were made. The images or other third party material in this article are included in the article's Creative Commons licence, unless indicated otherwise in a credit line to the material. If material is not included in the article's Creative Commons licence and your intended use is not permitted by statutory regulation or exceeds the permitted use, you will need to obtain permission directly from the copyright holder. To view a copy of this licence, visit http://creativecomm ons.org/licenses/by/4.0/.

\section{References}

1. Benosman M, Lum K (2008) Passive actuators' fault-tolerant control for affine nonlinear systems. Ifac Proc 41(2):14229-14234

2. Zhao K, Chen J (2018) Robust adaptive fault-tolerant control of nonlinear systems with assignable tracking performance under time-varying control coefficients. IEEE Access PP(99)(99):1

3. Guo W, Qiu A, Wen C (2019) Active actuator fault tolerant control based on generalized internal model control and performance compensation. IEEE Access 7:1

4. Hu H, Wang X, Chen L (2020) Impedance sliding mode control with adaptive fuzzy compensation for robot-environment interacting. IEEE Access PP(99): 1

5. Dong A, Du Z, Yan Z (2020) A sensorless interaction forces estimator for bilateral teleoperation system based on online sparse Gaussian process regression. Mech Mach Theory 143:103620

6. Pontryagin L (1959) Optimal control processes. Uspehi Mat-Nauk. 14:3-20

7. Bellman R, Dreyfus S (2013) Dynamic programming. Science 153(3731):34-37

8. Lewis F, Syrmos V (1995) Optimal control A Wiley-Interscience Pub

9. Abu-Khalaf M, Lewis F (2005) Nearly optimal control laws for nonlinear systems with saturating actuators using a neural network HJB approach. Automatica 41(5):779-791

10. Pan H, Xin M (2013) Nonlinear robust and optimal control of robot manipulators. Nonlinear Dyn 76(1):237-254

11. Lian C, Xu X, Chen H, He H (2017) Near-Optimal tracking control of mobile robots via receding-horizon dual heuristic programming. IEEE Trans Cybern 46(11):2484-2496

12. Dong N, Chen Z (2012) A novel ADP based model-free predictive control. Nonlinear Dyn 69(1-2):89-97

13. Luo B, Liu D, Wu H (2017) Adaptive constrained optimal control design for data-based nonlinear discrete-time systems with criticonly structure. IEEE Trans Neural Netw Learn Syst 1-13

14. Wei Q, Li B, Song R (2018) Discrete-time stable generalized selflearning optimal control with approximation errors. IEEE Trans Neural Netw Learn Syst 1(99):1-13

15. Liu C, Yu C, Gao B, Shah S, Tapus A (2021) Towards a balancing safety against performance approach in human-robot comanipulation for door-closing emergencies. Complex Intell Syst. $1-14$

كدينة الملك عبدالعزيز

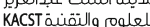

16. Zhao B, Jia L, Xia H, Li Y (2018) Adaptive dynamic programmingbased stabilization of nonlinear systems with unknown actuator saturation. Nonlinear Dyn 93(6):2089-2103

17. Zhu X, Ma B, Dong B, Liu K, Li Y (2020) Adaptive dynamic programming-based sliding mode optimal position-force control for reconfigurable manipulators with uncertain disturbance. In: 2020 Chinese Control and Decision Conference. IEEE.; August 22-24, Hefei. pp 421-427

18. Wei Q, Liu D (2014) Data-driven neuro-optimal temperature control of water-gas shift reaction using stable iterative adaptive dynamic programming. IEEE Trans Ind Electron 61(11):6399_ 6408

19. Biao L, Wu H, Huang T, Liu D (2014) Data-based approximate policy iteration for affine nonlinear continuous-time optimal control design. Automatica 50(12):3281-3290

20. Li C, Liu D, Wang D (2016) Data-based optimal control for weakly coupled nonlinear systems using policy iteration. IEEE Trans Syst Man Cybern B PP(99):1-11

21. Zhang H, Luo Y, Liu D (2009) Neural-network-based near-optimal control for a class of discrete-time affine nonlinear systems with control constraints. IEEE Trans Neural Netw 20(9):1490-1503

22. Liu C, Gao B, Zhao J, Shah S (2018) Orbitally stabilizing control for the underactuated translational oscillator with rotational actuator system: design and experimentation. Proc IMechE Part I: J Systems and Control Engineering, pp 1-10

23. Jiang Y, Jiang Z (2012) Robust Adaptive dynamic programming for large-scale systems with an application to multimachine power systems. IEEE Trans Circ Syst II-Express Briefs. 59(10):693-697

24. Wang D, Liu D, Li H (2014) Policy iteration algorithm for online design of robust control for a class of continuous-time nonlinear systems. IEEE Trans Autom Sci Eng 11(2):627-632

25. Wang D, Liu D, Li H, Luo B, Ma H (2016) An approximate optimal control approach for robust stabilization of a class of discretetime nonlinear systems with uncertainties. IEEE Trans Syst Man Cybern B 46(5):713-717

26. Zhao B, Liu D, Li Y (2016) Online fault compensation control based on policy iteration algorithm for a class of affine nonlinear systems with actuator failures. IET Contr Theory Appl 10(15):1816-1823

27. HeW Dong Y (2018) Adaptive fuzzy neural network control for a constrained robot using impedance learning. IEEE Trans Neural Netw Learn Syst PP(99):1-13

28. Roveda L, Pallucca G, Pedrocchi N, Braghin FTLM (2017) Iterative learning procedure with reinforcement for high-accuracy force tracking in robotized tasks. IEEE Trans Ind. Inf pp 1

29. Jia Q, Tang W (2018) Consensus of multi-agents with event-based nonlinear coupling over time-varying digraphs. IEEE Trans Circuits Syst II-Express. Briefs

30. Tong S, Shuai S, Li Y (2014) Adaptive fuzzy decentralized control for stochastic large-scale nonlinear systems with unknown deadzone and unmodeled dynamics. Neurocomputing 135(jul.5):367377

31. Zhao B, Li Y, Liu D (2017) Self-tuned local feedback gain based decentralized fault tolerant control for a class of large-scale nonlinear systems. Neurocomputing 235:147-156

32. Li Z, Melek W, Clark C (2009) Decentralized robust control of robot manipulators with harmonic drive transmission and application to modular and reconfigurable serial arms. Robotica 27(2):291-302

33. Bian T, Jiang Y, Jiang Z (2015) Decentralized adaptive optimal control of large-scale systems with application to power systems. IEEE Trans Ind Electron 62(4):2439-2447

34. Zhao B, Wang D, Shi G, Liu D, Li Y (2018) Decentralized control for large-scale nonlinear systems with unknown mismatched interconnections via policy iteration. IEEE Trans Syst Man Cybern B 48(10):1725-1735 
35. Zhao B, Li Y (2014) Local joint information based active fault tolerant control for reconfigurable manipulator. Nonlinear Dyn 77(3):859-876

36. Liu C, He J, Gao B (2019) Movement planning and control of an overhead power transmission line inspection bionic-robot. pp $25-29$
Publisher's Note Springer Nature remains neutral with regard to jurisdictional claims in published maps and institutional affiliations. 